\title{
LAGRANGE INTERPOLATION IN THE ZEROS OF BESSEL FUNCTIONS BY ENTIRE FUNCTIONS OF EXPONENTIAL TYPE AND MEAN CONVERGENCE
}

\author{
Georgi R. Grozev and Qazi I. Rahman
}

\begin{abstract}
Let $J_{\alpha}$ be the Bessel function of the first kind of order $\alpha>-1$. Then $G_{\alpha}(z):=z^{-\alpha} J_{\alpha}(z)$ is an entire function whose zeros are all real. We note that under appropriate conditions, the Lagrange interpolant of $f: \mathbb{R} \rightarrow \mathbb{C}$ in the zeros of $G_{\alpha}(\tau z)$ where $\tau>0$ is an entire function of exponential type $\tau$. We denote it by $L_{\tau, \alpha}(f ; z)$ and study the mean convergence of $L_{\tau, \alpha}(f ; \cdot)$ to $f$ as $\tau \rightarrow \infty$. We obtain a theorem which is analogous to two well-known results, one due to J. Marcinkiewicz and another due to R. Askey. Some of the lemmas which we need for our proof of the theorem are results of independent interest; for example, Lemma 13 is an extension of the Whittaker-Shannon sampling theorem.
\end{abstract}

\section{Introduction and statement of the main result}

For each $n \in \mathbb{N}$, let a system of $n$ distinct nodes

$$
-1 \leq x_{n, n}<\cdots<x_{n, k}<\cdots<x_{n, 1} \leq 1
$$

be specified. Let $L_{n-1}(f ; \cdot)$ denote the polynomial of degree $\leq n-1$ which interpolates $f$ at the given nodes. It was shown by Faber [10] that for some $f \in C[-1,1]$, the sequence $\left\{L_{n}(f ; x)\right\}$ does not converge uniformly to $f(x)$.

Let $Q_{0}, Q_{1}, \ldots$ be an orthogonal system of polynomials on $[-1,1]$ corresponding to some non-negative weight function $w$ belonging to $L^{1}[-1,1]$. It was proved by Erdös and Turán [9] that if the points in (1) are the zeros of $Q_{n}$, then for each $f \in C[-1,1]$ and $p=2$

$$
\int_{-1}^{1}\left|f(x)-L_{n}(f ; x)\right|^{p} w(x) d x \rightarrow 0 \quad \text { as } \quad n \rightarrow \infty .
$$

At about the same time as Erdös and Turán, but independently of them, Marcinkiewicz [19] proved the following

Theorem A. For each $n \in \mathbb{N}$, let

$$
\theta_{n, k}:=\frac{2 k \pi}{2 n+1} \quad k=0, \pm 1, \pm 2, \ldots, \pm n,
$$

and denote by $t_{n}(f ; \cdot)$ the trigonometric interpolatory polynomial of degree not exceeding $n$ with

$$
t_{n}\left(f ; \theta_{n, k}\right)=f\left(\theta_{n, k}\right)
$$

Received January 26, 1995, revised September 7, 1995.

1991 Mathematics Subject Classification. 30D10, 33C10, 41A05.

Key words and phrases: entire functions of exponential type, zeros of Bessel functions, Lagrange interpolation, mean convergence, Marcinkiewicz inequality, sampling theorems. 
If $f: \mathbb{R} \rightarrow \mathbb{C}$ is a continuous, $2 \pi$-periodic function, then for every $p>0$

$$
\lim _{n \rightarrow \infty} \int_{0}^{2 \pi}\left|f(\theta)-t_{n}(f ; \theta)\right|^{p} d \theta=0 .
$$

It may be added that $\lim \sup _{n \rightarrow \infty}\left|f(\theta)-t_{n}(f ; \theta)\right|=\infty$ for every $\theta$ if the continuous and $2 \pi$-periodic function $f$ is suitably chosen $[14,20]$.

Note that the case $w(x)=\left(1-x^{2}\right)^{-1 / 2}$ of (2) is contained in (3).

Askey [2,3] looked for values of $p>2$ for which (2) holds and observed that it was not possible to find such a $p$ for all weights. In the positive direction, he proved the following

Theorem B. [3, Theorem 10] Let $w(x):=(1-x)^{\alpha}(1+x)^{\beta}$ and $P_{n}^{\alpha, \beta}$ be the corresponding orthogonal (Jacobi) polynomial of degree $n$. If the points in (1) are the zeros of $P_{n}^{\alpha, \beta}$, then for each $f \in C[-1,1]$,

$$
\lim _{n \rightarrow \infty} \int_{-1}^{1}\left|f(x)-L_{n}(f ; x)\right|^{p}(1-x)^{\alpha}(1+x)^{\beta} d x=0
$$

for $p<4(\alpha+1) /(2 \alpha+1)$ when

(i) $\alpha, \beta>-1 / 2$, or

(ii) $|\alpha-k| \leq 1+\beta,-1<\beta<-\frac{1}{2}, 2 k=2,3, \ldots$.

For further results about the mean convergence of Lagrange interpolation based on the zeros of Jacobi polynomials, see [23] and some of the papers cited therein.

We consider Lagrange interpolation of non-periodic functions in an infinite set of points on $\mathbb{R}$. Polynomials or trigonometric polynomials are clearly not suitable for such a purpose. According to a well-known result, a complex-valued function $t$ is a trigonometric polynomial of degree $n$ if and only if it is the restriction to $\mathbb{R}$ of an entire function of exponential type $n$ which is periodic with period $2 \pi$. Thus, it is natural to use (non-periodic) entire functions of exponential type to interpolate non-periodic functions in an infinite set of points on $\mathbb{R}$. If so, what kind of points on $\mathbb{R}$ would be suitable for interpolation by entire functions of exponential type and for obtaining a convergence theorem like the one of Marcinkiewicz? In this paper, we shall see that multiples of the zeros of Bessel functions of the first kind of order $\alpha>-1$ are such points.

The Bessel function of the first kind of order $\alpha>-1$ can be defined by [33, p. 40]

$$
J_{\alpha}(z):=\left(\frac{1}{2} z\right)^{\alpha} \sum_{\nu=0}^{\infty}(-1)^{\nu} \frac{\left(\frac{1}{2} z\right)^{2 \nu}}{\nu ! \Gamma(\nu+\alpha+1)} .
$$

Note. Here and elsewhere in the text $\zeta^{\alpha}, \zeta \neq 0$ will mean $\exp (\alpha \log \zeta)$ where the logarithm has its principal value.

In view of (4), it is interesting to note that according to a classical formula [31, Theorem 8.1.1], if $\alpha, \beta$ are real, then

$$
\lim _{n \rightarrow \infty} n^{-\alpha} P_{n}^{\alpha, \beta}\left(\cos \frac{z}{n}\right)=\left(\frac{1}{2} z\right)^{-\alpha} J_{\alpha}(z)
$$

uniformly in every bounded region of the complex plane .

From the coefficients in the expansion (5) for $J_{\alpha}(z)$ it is easily seen that

$$
G_{\alpha}(z):=z^{-\alpha} J_{\alpha}(z)
$$


is an even entire function of order 1 type 1 [5, Theorem 2.2.10] and so is of exponential type 1 . It may be mentioned that

$$
G_{\alpha}(z)= \begin{cases}\sqrt{\frac{2}{\pi}} \cos z & \text { if } \alpha=-\frac{1}{2} \\ \sqrt{\frac{2}{\pi}} \frac{\sin z}{z} & \text { if } \alpha=\frac{1}{2}\end{cases}
$$

According to a theorem of Lommel [33, p. 482], the function $J_{\alpha}(z)$ has only real zeros if $\alpha>-1$. They are all simple with the possible exception of $z=0$. Arranging the positive zeros of $J_{\alpha}$ in increasing order of magnitude, we shall denote the $k$ th zero by $j_{\alpha, k}$ or simply by $j_{k}$, if there is no ambiguity. For each $k \in \mathbb{N}$, the zero $-j_{\alpha, k}$ of $J_{\alpha}$ will be denoted by $j_{\alpha,-k}$ or by $j_{-k}$.

From (6) it follows that if $-1<x_{n, n}<\cdots<x_{n, 1}<1$ are the zeros of $P_{n}^{\alpha, \beta}$, and if we write $x_{n, k}=\cos \theta_{n, k}, 0<\theta_{n, k}<\pi$, then for a fixed $k \geq 1$

$$
\theta_{n, k} \sim \frac{j_{k}}{n} \quad \text { as } n \rightarrow \infty \text {. }
$$

To $f: \mathbb{R} \rightarrow \mathbb{C}$, we formally associate

$$
L_{\tau, \alpha}(f ; z)=\sum_{\substack{\nu=-\infty \\ \nu \neq 0}}^{\infty} \frac{G_{\alpha}(\tau z)}{G_{\alpha}^{\prime}\left(j_{\nu}\right)\left(\tau z-j_{\nu}\right)} f\left(\frac{j_{\nu}}{\tau}\right) \quad \tau>0
$$

which interpolates $f$ at the points $j_{k} / \tau,(k \in \mathbb{Z} \backslash\{0\})$. Note that $\tau$ does not have to be an integer.

In order to state our main theorem, we need to introduce a couple of definitions.

Definition 1. Given $p>1$, we denote by $\mathcal{F}^{\alpha, p}(\delta)$ the set of all measurable functions $f: \mathbb{R} \rightarrow \mathbb{C}$ satisfying

$$
f(x)=O\left(1 /(|x|+1)^{\alpha+\frac{1}{2}+\frac{1}{p}+\delta}\right) \quad x \in \mathbb{R},
$$

for some $\delta>0$ and by $\mathcal{F}^{\alpha, p}$ the union $\bigcup_{\delta>0} \mathcal{F}^{\alpha, p}(\delta)$. It is clear that if $f \in \mathcal{F}^{\alpha, p}$, then $|x|^{\alpha+\frac{1}{2}} f(x) \in L^{p}(\mathbb{R})$ for all $p>1$ when $\alpha \geq-\frac{1}{2}$ and for $1<p<\frac{2}{|2 \alpha+1|}$ when $-1<\alpha<-1 / 2$.

Definition 2. Let $\mathcal{R}$ be the set of all functions $f: \mathbb{R} \rightarrow \mathbb{C}$ which are Riemann integrable on every finite interval.

Our analogue of Theorem A now may be stated as follows.

Theorem 1. Let $\alpha \geq-1 / 2, p>1$ or $-1<\alpha<-1 / 2,1<p<\frac{2}{|2 \alpha+1|}$. Then

$$
\left\|f-L_{\tau, \alpha}(f ; \cdot)\right\|_{\alpha, p}:=\left(\int_{-\infty}^{\infty}\left|x^{\alpha+\frac{1}{2}}\left(f(x)-L_{\tau, \alpha}(f ; x)\right)\right|^{p} d x\right)^{1 / p} \rightarrow 0 \quad \text { as } \tau \rightarrow \infty
$$

if $f \in \mathcal{F}^{\alpha, p} \bigcap \mathcal{R}$.

Note that the integral of $\left|x^{\alpha+\frac{1}{2}}\left(f(x)-L_{\tau, \alpha}(f ; x)\right)\right|^{p}$ over $(-1,1)$, and so a fortiori over $(-\infty, \infty)$, may not be defined if $\alpha \in(-1,-1 / 2)$ and $p \geq \frac{2}{|2 \alpha+1|}$.

Remark 1. We shall show that if $\alpha \geq-1 / 2$, then

$$
\sup _{x \in \mathbb{R}}\left|x^{\alpha+\frac{1}{2}}\left(f(x)-L_{\tau, \alpha}(f ; x)\right)\right|
$$

may not tend to zero as $\tau \rightarrow \infty$ if $f$ satisfies (8). 
Remark 2. The case $\alpha=-1 / 2$ of Theorem 1 was treated in [27].

Remark 3. In the case $\alpha \geq-1 / 2$, we may replace (9) by

$$
\int_{-\infty}^{\infty}\left|f(x)-L_{\tau, \alpha}(f ; x)\right|^{p}|x|^{2 \alpha+1} d x \rightarrow 0 \quad \text { as } \tau \rightarrow \infty
$$

if $p \geq 2$.

1.1. Why the weight $|x|^{2 \alpha+1}$ in $\left(\mathbf{9}^{\prime}\right)$ ? Let $\left\{\lambda_{n}\right\}$ be an increasing sequence of real numbers, $\lambda_{n} \neq 0, \lambda_{-n}=-\lambda_{n}$, and for large $n, 0<\lambda_{n} \leq n+\alpha / 2-1 / 2$. According to a result of Boas and Pollard ([6] or [5, Corollary 9.6.14]), if $f$ is an entire function of exponential type satisfying

$$
|y|^{\beta} f(x+i y) e^{-\pi|y|} \rightarrow 0, \quad|y| \rightarrow \infty
$$

and $\alpha \leq \beta$, then $f(z) \equiv 0$ if $f\left(\lambda_{n}\right)=0$ for $n= \pm 1, \pm 2, \ldots$.

Using some of the facts about the zeros $j_{\alpha, k}$, which are listed in the next section, it easily can be concluded from the preceding result of Boas and Pollard that if an entire function of exponential type $\sigma<\tau$ vanishes at the zeros of $G_{\alpha}(\tau z)$, then it must be identically zero; here $\sigma=\tau$ is inadmissible as the example $G_{\alpha}(\tau z)$ shows. This means that an entire function of exponential type $\sigma$ is completely determined by its values at the points $j_{\alpha, k} / \tau$ if $\sigma<\tau$, but not necessarily so if $\sigma=\tau$. However, the quadrature formula $[11,13]$

$$
\int_{-\infty}^{\infty}|x|^{2 \alpha+1} f(x) d x=\frac{2}{\tau^{2 \alpha+2}} \sum_{\substack{k=-\infty \\ k \neq 0}}^{\infty}\left|\frac{1}{G_{\alpha}^{\prime}\left(\left|j_{\alpha, k}\right|\right)}\right|^{2} f\left(\frac{j_{\alpha, k}}{\tau}\right)
$$

holds for all entire functions of exponential type $2 \tau$ if the integral on the left exists in the sense of Lebesgue.

Since (10) correctly evaluates the integral for all entire functions of (exponential) type 2 times $\tau$, we see it as a Gaussian quadrature formula. We find it interesting that the weight $|x|^{2 \alpha+1}$ and the nodes $j_{\alpha, k} / \tau$ involved in (10) play the same role in $\left(9^{\prime}\right)$ as the corresponding quantities $(1-x)^{\alpha}(1+x)^{\beta}$ and the zeros of $P_{n}^{\alpha, \beta}$, involved in the Gauss-Jacobi quadrature formula, do in (4). It is for this reason that we consider $\left(9^{\prime}\right)$ to be an analogue of (4).

\section{Auxiliary results}

2.1. Relevant facts about Bessel functions and their zeros. The asymptotic formula [35, p. 368]

$$
\begin{aligned}
J_{\alpha}(z)= & \left(\frac{2}{\pi z}\right)^{1 / 2}\left\{\left(\cos \left(z-\frac{\alpha \pi}{2}-\frac{\pi}{4}\right)\right)\left(1+O\left(z^{-2}\right)\right)\right. \\
& \left.+\left(\sin \left(z-\frac{\alpha \pi}{2}-\frac{\pi}{4}\right)\right) O\left(z^{-1}\right)\right\}
\end{aligned}
$$

holds for $|z|$ large and $|\arg z|<\pi$. From (11) follows the auxiliary result [11, Lemma 1]

Lemma 1. There exists a positive constant $c_{1}=c_{1}(\alpha)$ depending only on $\alpha$ such that for all large $R$ of the form $\left(N+\frac{\alpha}{2}+\frac{1}{4}\right) \pi$ where $N \in \mathbb{N}$, we have

$$
\left|J_{\alpha}\left(R e^{i \theta}\right)\right|>c_{1} \frac{1}{\sqrt{R}} \cdot e^{R|\sin \theta|}, \quad \theta \in \mathbb{R} .
$$


Since $J_{\alpha}(z)-\left(\frac{2}{\pi z}\right)^{1 / 2} \cos \left(z-\alpha \frac{\pi}{2}-\frac{\pi}{4}\right)$ is regular and of exponential type in $\mathfrak{R e} z>0$ and is $O\left(x^{-3 / 2}\right)$ as $x \rightarrow \infty$ by (11), it follows from Lemma 2 below that

$$
J_{\alpha}^{\prime}(x)=-\left(\frac{2}{\pi x}\right)^{\frac{1}{2}} \sin \left(x-\frac{\alpha \pi}{2}-\frac{\pi}{4}\right)+O\left(x^{-3 / 2}\right) \quad \text { as } \quad x \rightarrow \infty .
$$

Lemma 2. [15, p. 198, Lemma 14] If $f$ is of exponential type in $\mathfrak{R e} z>0$, and if for some $\lambda \in \mathbb{R}$,

$$
f(x)=O\left(x^{\lambda}\right) \quad \text { as } x \rightarrow \infty
$$

then

$$
f^{\prime}(x)=O\left(x^{\lambda}\right) \quad \text { as } x \rightarrow \infty .
$$

For all large $m \in \mathbb{N}$, the number of zeros of $J_{\alpha}$ in the interval $\left(0, m \pi+\left(\frac{\alpha}{2}+\frac{1}{4}\right) \pi\right)$ is exactly $m$ [33, p. 497].

According to a result of McMahon ([22]; also see [21], [33, p. 506]) the $k$ th positive zero is given by

$$
\begin{aligned}
j_{\alpha, k}=( & \left.+\frac{1}{2} \alpha-\frac{1}{4}\right) \pi-\frac{4 \alpha^{2}-1}{8 \pi\left(k+\frac{1}{2} \alpha-\frac{1}{4}\right)}-\frac{\left(4 \alpha^{2}-1\right)\left(28 \alpha^{2}-31\right)}{384 \pi^{3}\left(k+\frac{1}{2} \alpha-\frac{1}{4}\right)^{3}} \\
& -\frac{\left(4 \alpha^{2}-1\right)\left(1328 \alpha^{4}-3928 \alpha^{2}+3779\right)}{15360 \pi^{5}\left(k+\frac{1}{2} \alpha-\frac{1}{4}\right)^{5}}-\cdots .
\end{aligned}
$$

Thus, $j_{\alpha, k+1}-j_{\alpha, k} \sim \pi$ for all large $k$. Since for all $k \in \mathbb{Z} \backslash\{0\}$ the zeros $j_{\alpha, k}$ are simple, it follows that if $j_{0}:=0$, then

$$
\begin{aligned}
& j_{\alpha, k+1}-j_{\alpha, k} \geq \delta_{1}=\delta_{1}(\alpha), \\
& j_{\alpha, k+1}-j_{\alpha, k} \leq \delta_{2}=\delta_{2}(\alpha),
\end{aligned}
$$

where $\delta_{1}, \delta_{2}$ are positive constants depending only on $\alpha$.

Formula (14) implies that for all large $k$

$$
\left|\sin \left(j_{k}-\frac{\alpha \pi}{2}-\frac{\pi}{4}\right)\right|>\frac{1}{\sqrt{2}} .
$$

Hence, by (13), there exists $k_{0} \in \mathbb{N}$ such that

$$
\left|J_{\alpha}^{\prime}\left(\left|j_{k}\right|\right)\right|>\frac{1}{2 \sqrt{\pi}\left|j_{k}\right|^{\frac{1}{2}}} \quad \text { if }|k| \geq k_{0} .
$$

The zeros of $J_{\alpha}$ are all simple and so $J_{\alpha}^{\prime}\left(\left|j_{k}\right|\right) \neq 0$ for all $k \neq 0$. Therefore, from (17), it follows that for some positive constant $c_{2}=c_{2}(\alpha)$ depending only on $\alpha$, we have

$$
\left|J_{\alpha}^{\prime}\left(\left|j_{k}\right|\right)\right|>\frac{c_{2}}{\left|j_{k}\right|^{\frac{1}{2}}} \quad k= \pm 1, \pm 2, \ldots
$$

and so

$$
\left|G_{\alpha}^{\prime}\left(j_{k}\right)\right|=\frac{\left|J_{\alpha}^{\prime}\left(\left|j_{k}\right|\right)\right|}{\left|j_{k}\right|^{\alpha}}>\frac{c_{2}}{\left|j_{k}\right|^{\alpha+\frac{1}{2}}} \quad k= \pm 1, \pm 2, \ldots
$$

In addition, we shall need

Lemma 3. For all $\alpha>-1$,

$$
\int_{0}^{\infty} \frac{G_{\alpha}(x)}{x^{2}-j_{1}^{2}} d x \neq 0
$$


Proof. According to a known formula ([1, formula 11.4.5] or [33, pp. 426-427, formula (11)]), we have

$$
\int_{0}^{\infty} \frac{G_{\alpha}(x)}{x^{2}+\zeta^{2}} d x=\frac{\pi}{2 \zeta^{\alpha+1}}\left\{I_{\alpha}(\zeta)-\mathrm{L}_{\alpha}(\zeta)\right\}, \quad \alpha>-\frac{5}{2}, \quad \mathfrak{R e} \zeta>0,
$$

where $I_{\alpha}$ is related to $J_{\alpha}$ by $([1$, formula 9.6 .3$]$ or $[33$, p. 77 , formula (2)])

$$
I_{\alpha}(z)=i^{-\alpha} J_{\alpha}(i z),
$$

and $\mathrm{L}_{\alpha}$ to the Struve function $([1$, p. 496$]$ or $[33$, p. 328$])$

$$
\mathrm{H}_{\alpha}(z)=\left(\frac{1}{2} z\right)^{\alpha+1} \sum_{k=0}^{\infty} \frac{(-1)^{k}\left(\frac{1}{2} z\right)^{2 k}}{\Gamma\left(k+\frac{3}{2}\right) \Gamma\left(k+\alpha+\frac{3}{2}\right)}
$$

by the formula ([1, formula 12.2 .1$]$ or $[33$, p. 329 , formula $(11)]$ )

$$
\mathrm{L}_{\alpha}(z)=i^{-\alpha-1} \mathrm{H}_{\alpha}(i z) \text {. }
$$

It is clear that if $\zeta=i j_{1}+\xi$ where $\xi>0$, then

$$
\frac{\pi}{2 \zeta^{\alpha+1}}\left(I_{\alpha}(\zeta)-\mathrm{L}_{\alpha}(\zeta)\right) \rightarrow \frac{\pi}{2\left(i j_{1}\right)^{\alpha+1}}\left(I_{\alpha}\left(i j_{1}\right)-\mathrm{L}_{\alpha}\left(i j_{1}\right)\right) \quad \text { as } \xi \rightarrow 0 \text {. }
$$

Besides, it is easily seen that as $\xi \rightarrow 0$,

$$
\int_{0}^{\infty} \frac{G_{\alpha}(x)}{x^{2}+\zeta^{2}} d x \rightarrow \int_{0}^{\infty} \frac{G_{\alpha}(x)}{x^{2}-j_{1}^{2}} d x
$$

In order to prove this, we note that

$$
\begin{aligned}
\int_{0}^{\infty} \frac{G_{\alpha}(x)}{x^{2}+\left(i j_{1}+\xi\right)^{2}} d x-\int_{0}^{\infty} \frac{G_{\alpha}(x)}{x^{2}-j_{1}^{2}} d x \\
=\int_{0}^{\infty} \frac{1}{x+j_{1}-i \xi} \cdot \frac{G_{\alpha}(x)}{x-j_{1}+i \xi} d x-\int_{0}^{\infty} \frac{G_{\alpha}(x)}{x^{2}-j_{1}^{2}} d x
\end{aligned}
$$

Let $\varepsilon>0$ be given. There exists a $\delta_{3}>0$ such that for all $\delta \leq \delta_{3}$,

$$
\left|\int_{j_{1}-\delta}^{j_{1}+\delta} \frac{G_{\alpha}(x)}{x^{2}-j_{1}^{2}} d x\right|<\frac{\varepsilon}{3} .
$$

Since $G_{\alpha}$ is an entire function, in the neighbourhood of $j_{1}$, we have

$$
G_{\alpha}(z)=a_{1}\left(z-j_{1}\right)+O\left(\left|z-j_{1}\right|^{2}\right), \quad a_{1}:=G_{\alpha}^{\prime}\left(j_{1}\right),
$$

so that for all real $\xi$

$$
\frac{\left|G_{\alpha}(x)\right|}{\sqrt{\left(x-j_{1}\right)^{2}+\xi^{2}}} \leq\left|a_{1}\right|+O\left(\left|x-j_{1}\right|\right) .
$$

Hence, there exists a $\delta_{4}>0$ such that for all $\delta \leq \delta_{4}$,

$$
\left|\int_{j_{1}-\delta}^{j_{1}+\delta} \frac{1}{x+j_{1}-i \xi} \frac{G_{\alpha}(x)}{x-j_{1}+i \xi} d x\right| \leq \int_{j_{1}-\delta}^{j_{1}+\delta} \frac{1}{\left|x+j_{1}\right|} \frac{\left|G_{\alpha}(x)\right|}{\sqrt{\left(x-j_{1}\right)^{2}+\xi^{2}}} d x<\frac{\varepsilon}{3} .
$$


Furthermore,

$$
\begin{aligned}
\int_{[0, \infty) \backslash\left(j_{1}-\delta, j_{1}+\delta\right)} & \left|\frac{G_{\alpha}(x)}{x^{2}-j_{1}^{2}}-\frac{G_{\alpha}(x)}{x^{2}+\zeta^{2}}\right| d x \\
& =\xi \int_{[0, \infty) \backslash\left(j_{1}-\delta, j_{1}+\delta\right)}\left|\frac{G_{\alpha}(x)}{x^{2}-j_{1}^{2}}\right| \cdot\left|\frac{\xi+2 i j_{1}}{x^{2}-j_{1}^{2}+\xi^{2}+2 i \xi j_{1}}\right| d x \\
& \leq \frac{\xi\left(\xi+2 j_{1}\right)}{\delta^{2}} \int_{[0, \infty) \backslash\left(j_{1}-\delta, j_{1}+\delta\right)}\left|\frac{G_{\alpha}(x)}{x^{2}-j_{1}^{2}}\right| d x \\
& <\frac{\varepsilon}{3}
\end{aligned}
$$

if $\delta:=\min \left\{\delta_{3}, \delta_{4}\right\}$ and $\xi$ is sufficiently small. The estimates (24), (25), and (26) readily imply (23). Hence, (20) holds also for $\zeta=i j_{1}$, i.e.,

$$
\int_{0}^{\infty} \frac{G_{\alpha}(x)}{x^{2}-j_{1}^{2}} d x=\frac{\pi}{2\left(i j_{1}\right)^{\alpha+1}}\left(I_{\alpha}\left(i j_{1}\right)-\mathrm{L}_{\alpha}\left(i j_{1}\right)\right) .
$$

According to (21) and (22), respectively, we have

$$
I_{\alpha}\left(i j_{1}\right)=i^{-\alpha} J_{\alpha}\left(j_{1} e^{\pi i}\right)=0 \text { and } \mathrm{L}_{\alpha}\left(i j_{1}\right)=i^{-\alpha-1} \mathrm{H}_{\alpha}\left(j_{1} e^{\pi i}\right) .
$$

Consequently,

$$
\int_{0}^{\infty} \frac{G_{\alpha}(x)}{x^{2}-j_{1}^{2}} d x=\frac{(-1)^{-\alpha} \pi}{2 j_{1}^{\alpha+1}} \mathrm{H}_{\alpha}\left(j_{1} e^{\pi i}\right) .
$$

Hence, the Lemma holds if and only if

$$
\mathrm{H}_{\alpha}\left(j_{1} e^{\pi i}\right) \neq 0 \text {. }
$$

We shall show that (27) is indeed true.

Case 1. $-1<\alpha \leq-\frac{1}{2}$.

In this case, $0<j_{1} \leq \frac{\pi}{2}$. According to a known formula [33, p. 328]

$$
\mathrm{H}_{\alpha}(z)=\frac{\left(\frac{1}{2} z\right)^{\alpha+1}}{\Gamma\left(\frac{3}{2}\right) \Gamma\left(\alpha+\frac{3}{2}\right)}(1+\theta(z))
$$

where

$$
|\theta(z)|<\frac{2}{3} \exp \left\{\frac{\frac{1}{4}|z|^{2}}{\left|\alpha+\frac{3}{2}\right|}-1\right\}
$$

Thus,

$$
\left|\theta\left(-j_{1}\right)\right|<\frac{2}{3} \exp \left\{\frac{\frac{1}{4}\left|j_{1}\right|^{2}}{\left|\alpha+\frac{3}{2}\right|}-1\right\} \leq \frac{2}{3} \exp \left(\frac{\pi^{2}}{8}-1\right)<0.8422,
$$

i.e., $\left|1+\theta\left(-j_{1}\right)\right|>0.1588$ and so $\mathrm{H}_{\alpha}\left(j_{1} e^{\pi i}\right) \neq 0$.

Case 2. $-\frac{1}{2}<\alpha<\frac{1}{2}$.

In this case, $\frac{\pi}{2}<j_{1}<\pi$ and ([1, formula 12.1.6] or [33, p. 328, formula (1)])

$$
\mathrm{H}_{\alpha}\left(j_{1} e^{\pi i}\right)=-\frac{2\left(-\frac{1}{2} j_{1}\right)^{\alpha}}{\Gamma\left(\alpha+\frac{1}{2}\right) \Gamma\left(\frac{1}{2}\right)} \int_{0}^{1}\left(1-t^{2}\right)^{\alpha-\frac{1}{2}} \sin \left(j_{1} t\right) d t .
$$

Since $\sin \left(j_{1} t\right)>0$ for $0<t<1$, the integral is (strictly) positive and so $\mathrm{H}_{\alpha}\left(j_{1} e^{\pi i}\right) \neq 0$.

Case 3. $\alpha \geq \frac{1}{2}$.

It is known [33, Section 10.45] that

$$
\mathrm{H}_{\alpha}(x)>0 \quad \text { for } x>0 \text {. }
$$


Therefore,

$$
\mathrm{H}_{\alpha}^{*}(x):=\frac{\mathrm{H}_{\alpha}(x)}{\left(\frac{1}{2} x\right)^{\alpha+1}}>0 \quad \text { for } x \geq 0 .
$$

However, $\mathrm{H}_{\alpha}^{*}$ is an even function, so $\mathrm{H}_{\alpha}^{*}(x)>0$ for all $x \in \mathbb{R}$. In particular, $\mathrm{H}_{\alpha}^{*}\left(j_{1} e^{\pi i}\right)>0$ and

$$
\mathrm{H}_{\alpha}\left(j_{1} e^{\pi i}\right)=\left(-\frac{1}{2} j_{1}\right)^{\alpha+1} \mathrm{H}_{\alpha}^{*}\left(j_{1} e^{\pi i}\right) \neq 0
$$

2.2. Relevant facts about functions of exponential type. In addition to Lemma 2 , we shall need some other facts about functions of exponential type. They are contained in Lemmas 4-9.

Lemma 4. If $g$ is regular and of exponential type for $x>0$ and $\int_{0}^{\infty}|g(x)|^{p} d x<\infty$ for some positive $p$, then [4]

$$
\int_{0}^{\infty}|g(x+i y)|^{p} d x<\infty
$$

for every real $y$, and $g(x) \rightarrow 0$ as $x \rightarrow \infty$. In case $g$ is an entire function of exponential type $\tau$ and $\int_{-\infty}^{\infty}|g(x)|^{p} d x<\infty, p>0$, then ([25] or [5, Theorem 6.7.1])

$$
\left(\int_{-\infty}^{\infty}|g(x+i y)|^{p} d x\right)^{1 / p} \leq e^{\tau|y|}\left(\int_{-\infty}^{\infty}|g(x)|^{p} d x\right)^{1 / p} .
$$

Lemma 5. [4], [13, Lemma 1] Let $\left\{\lambda_{k}\right\}$ be an increasing sequence of positive numbers with $\lambda_{k+1}-\lambda_{k} \geq \delta>0$. If $g$ is regular and of exponential type in the open right half-plane such that

$$
\int_{0}^{\infty}|g(x)| d x<\infty
$$

then

$$
\sum_{k=1}^{\infty}\left|g\left(\lambda_{k}\right)\right|<\infty
$$

Lemma 6. [5, Theorem 5.6.8], [15, Lemma 9] Let $g(z)$ be regular and of exponential type $<\pi,|\arg z| \leq \beta \leq \frac{\pi}{2}, h(0):=\limsup _{x \rightarrow \infty} \frac{\log |g(x)|}{x} \leq 0$. Then we can write $g(z)=g_{1}(z)+g_{2}(z)$ where $g_{1}(z)$ is an entire function of exponential type less than $\pi$ such that $g_{1}(x)=O(1 /|x|)$ as $x \rightarrow-\infty$, while $g_{2}(z)$ is regular and of exponential type in $|\arg z| \leq \beta$ satisfying $g_{2}(x)=O(1 / x)$ as $x \rightarrow+\infty$.

The preceding result is really due to Macintyre [18], although it was not stated in this form by him.

Remark 4. The restriction on the exponential type of $g$ is redundant. Indeed, if $g$ is of exponential type $\tau \geq \pi$, then $g\left(\frac{\pi}{2 \tau} z\right)$ is of exponential type $\frac{\pi}{2}$.

Lemma 7. If $g$ is an entire function of exponential type $\tau$, and if $g \in L^{p}(\mathbb{R}), p>0$, then

$$
\left(\int_{-\infty}^{\infty}\left|g^{\prime}(x)\right|^{p} d x\right)^{1 / p} \leq \tau\left(\int_{-\infty}^{\infty}|g(x)|^{p} d x\right)^{1 / p}
$$


The case $p \geq 1$ of this inequality is classical [5, Theorem 11.3.3]. That it holds also for $0<p<1$ was proved in [26].

Lemma 8. If $g$ is holomorphic and of exponential type in the open right half-plane and belongs to $L^{p}(0, \infty)$ for some $p>1$, then $g^{\prime} \in L^{p}[\varepsilon, \infty)$ for every $\varepsilon>0$.

Proof. Since $g \in L^{p}(0, \infty), g(x) \rightarrow 0$ as $x \rightarrow \infty$ by Lemma 4 and so, in particular, $h(0) \leq 0$. By Lemma 6 applied to $g(z+\varepsilon), g(z)=g_{1}(z)+g_{2}(z)$ where $g_{1}$ is an entire function such that $g_{1}(x)=O(1 /|x|)$ as $x \rightarrow-\infty$ and $g_{2}$ is regular and of exponential type in $\mathfrak{R e} z \geq \varepsilon$ satisfying $g_{2}(x)=O(1 / x)$ as $x \rightarrow+\infty$. Hence, $g_{1} \in L^{p}(-\infty, \varepsilon]$, $g_{2} \in L^{p}[\varepsilon, \infty)$. Since $g \in L^{p}(0, \infty)$, by hypothesis, $g_{1}=g-g_{2}$ belongs to $L^{p}[\varepsilon, \infty)$, so $g_{1} \in L^{p}(\mathbb{R})$. By Lemma $7, g_{1}^{\prime}$ belongs to $L^{p}(\mathbb{R})$. Next, we note that the function $z g_{2}(z)$, which is of exponential type in $\mathfrak{R e} z \geq \varepsilon$, is bounded on $[\varepsilon, \infty)$. Hence, by Lemma 2 , its derivative $x g_{2}^{\prime}(x)+g_{2}(x)$ is also bounded on $[\varepsilon, \infty)$, so $g_{2}^{\prime}(x)=O(1 / x)$ as $x \rightarrow+\infty$; in particular, $g_{2}^{\prime} \in L^{p}[\varepsilon, \infty)$. It follows that $g^{\prime}=g_{1}^{\prime}+g_{2}^{\prime} \in L^{p}[\varepsilon, \infty)$.

We also need the following result due to Lindelöf.

Lemma 9. [16, Theorem 18.3.5] Let $g$ be holomorphic and bounded in the upper halfplane. If $g$ is continuous at all finite points of the real axis, and $g(x) \rightarrow a$ when $x \rightarrow+\infty$, then

$$
\lim _{z \rightarrow \infty} g(z)=a
$$

uniformly in any sector $0 \leq \arg z \leq \pi-\delta, 0<\delta$.

2.3. Additional lemmas. The next two lemmas play an important role in our work.

Lemma 10. [32, Theorem A] Let $\left\{a_{k}\right\}_{k \in \mathbb{Z}}$ denote any sequence of numbers such that the series

$$
\sum_{k=-\infty}^{\infty}\left|a_{k}\right|^{p} \quad p>1
$$

is convergent, and let

$$
b_{n}:=\frac{1}{\pi} \sum_{k=-\infty}^{\infty} \frac{a_{k}}{k+n+\frac{1}{2}}
$$

for all values of $n$. Then, the series $\sum\left|b_{n}\right|^{p}$ is also convergent, and there is a number $N_{p}$ depending only on $p$ such that

$$
\sum_{n=-\infty}^{\infty}\left|b_{n}\right|^{p} \leq N_{p}^{p} \sum_{n=-\infty}^{\infty}\left|a_{n}\right|^{p} .
$$

Lemma 11. [25, p. 135 (Lemma 7)] Let $\left\{u_{k}\right\}_{k \in \mathbb{Z}}$ denote any sequence of numbers such that the series

$$
\sum_{k=-\infty}^{\infty}\left|u_{k}\right|^{p} \quad p>1
$$

is convergent, and let $\left\{t_{\mu}\right\}_{\mu \in \mathbb{Z}}$ be a sequence of positive numbers such that $t:=$ $\sum_{\mu=-\infty}^{\infty} t_{\mu}<\infty$. If

$$
v_{n}:=\sum_{k=-\infty}^{\infty} t_{n-k}\left|u_{k}\right|
$$


for all values of $n$, then

$$
\sum_{n=-\infty}^{\infty} v_{n}^{p} \leq t^{p} \sum_{k=-\infty}^{\infty}\left|u_{k}\right|^{p}
$$

\section{Proof of the main result}

\subsection{Preparatory lemmas.}

3.1.1. Properties of the interpolant $L_{\tau, \alpha}(f ; \cdot)$.

Lemma 12. Let $\alpha>-1, p>1, \tau>0$. If

$$
\sum_{\substack{k=-\infty \\ k \neq 0}}^{\infty}\left|\left(\frac{j_{k}}{\tau}\right)^{\alpha+\frac{1}{2}} f\left(\frac{j_{k}}{\tau}\right)\right|^{p}<\infty
$$

then the series for $L_{\tau, \alpha}(f ; z)$ converges absolutely and uniformly on all compact subsets of $\mathbb{C}$ and defines an entire function of exponential type $\tau$.

Proof. Let $E$ be an arbitrary compact subset of $\mathbb{C}$ and $N_{E}$ the smallest integer such that $|z| \leq \frac{1}{\tau} j_{N_{E}}$ for all $z \in E$. For $z \in E, N_{1}, N_{2} \in \mathbb{Z}, N_{1}<N_{2}$, we have

$$
\left|\sum_{\substack{k=N_{1} \\ k \neq 0}}^{N_{2}} \frac{G_{\alpha}(\tau z)}{G_{\alpha}^{\prime}\left(j_{k}\right)\left(\tau z-j_{k}\right)} f\left(\frac{j_{k}}{\tau}\right)\right| \leq A\left(N_{1}, N_{2}\right) \cdot B\left(N_{1}, N_{2}, z\right)
$$

where

$$
\begin{gathered}
A\left(N_{1}, N_{2}\right):=\left(\sum_{\substack{k=N_{1} \\
k \neq 0}}^{N_{2}}\left|\frac{f\left(j_{k} / \tau\right)}{G_{\alpha}^{\prime}\left(j_{k}\right)}\right|^{p}\right)^{1 / p}, \\
B\left(N_{1}, N_{2}, z\right):=\left(\sum_{\substack{k=N_{1} \\
k \neq 0}}^{N_{2}}\left|\frac{G_{\alpha}(\tau z)}{\tau z-j_{k}}\right|^{q}\right)^{1 / q}, \quad q=\frac{p}{p-1} .
\end{gathered}
$$

By (19)

$$
A\left(N_{1}, N_{2}\right) \leq \frac{1}{c_{2}}\left(\left.\left.\sum_{\substack{k=N_{1} \\ k \neq 0}}^{N_{2}}|| j_{k}\right|^{\alpha+\frac{1}{2}}\left|f\left(\frac{j_{k}}{\tau}\right)\right|\right|^{p}\right)^{1 / p}
$$

Clearly,

$$
B\left(N_{1}, N_{2}, z\right) \leq\left(\left(\sum_{\substack{|k| \leq N_{E} \\ k \neq 0}}+\sum_{N_{E}+1 \leq|k|<\infty}\right)\left|\frac{G_{\alpha}(\tau z)}{\tau z-j_{k}}\right|^{q}\right)^{1 / q} .
$$

The first sum on the right-hand side of (42) may be estimated as follows. Let $\delta_{1}$ be as in (15) and draw circles of radius $\frac{\delta_{1}}{3 \tau}$ around each of the points $\frac{1}{\tau} j_{l},|l| \leq N_{E}, l \neq 0$. On each such circle $\left|z-\frac{1}{\tau} j_{l}\right|=\frac{\delta_{1}}{3 \tau}$, we have

$$
\begin{aligned}
\left|\frac{G_{\alpha}(\tau z)}{\tau z-j_{k}}\right| & \leq \frac{3}{\delta_{1}} \max _{\left|z-\frac{1}{\tau} j_{l}\right|=\frac{\delta_{1}}{3 \tau}}\left|G_{\alpha}(\tau z)\right| \\
& \leq \frac{3}{\delta_{1}} \max _{|z| \leq \frac{\left(j_{N_{E}}+\frac{\left.\delta_{1}\right)}{3}\right)}{\tau}}\left|G_{\alpha}(\tau z)\right|=: \frac{3 M_{E}}{\delta_{1}} .
\end{aligned}
$$


Since $\frac{G_{\alpha}(\tau z)}{\tau z-j_{k}}$ is an entire function, it follows that the preceding estimate also holds inside each of the circles. If $z \in E$ but does not belong to any of the disks $\left|z-\frac{1}{\tau} j_{l}\right| \leq \frac{\delta_{1}}{3 \tau}$, $|l| \leq N_{E}, l \neq 0$, then $\left|\tau z-j_{k}\right|>\frac{\delta_{1}}{3}$ and the estimate (43) holds trivially. Hence, for all $z \in E$

$$
\sum_{\substack{|k| \leq N_{E} \\ k \neq 0}}\left|\frac{G_{\alpha}(\tau z)}{\tau z-j_{k}}\right|^{q} \leq\left(2 N_{E}\right)\left(\frac{3 M_{E}}{\delta_{1}}\right)^{q}
$$

Further, for $z \in E$

$$
\begin{aligned}
& \sum_{N_{E}+1 \leq|k|<\infty}\left|\frac{G_{\alpha}(\tau z)}{\tau z-j_{k}}\right|^{q} \leq\left(M_{E}\right)^{q} \sum_{N_{E}+1 \leq|k|<\infty} \frac{1}{\left(\left|j_{k}\right|-\left|j_{N_{E}}\right|\right)^{q}} \\
& \leq\left(M_{E}\right)^{q} \sum_{N_{E}+1 \leq|k|<\infty} \frac{1}{\left(|k|-N_{E}\right)^{q} \delta_{1}^{q}} \\
& <\left(\frac{M_{E}}{\delta_{1}}\right)^{q} \sum_{\substack{k=-\infty \\
k \neq 0}}^{\infty} \frac{1}{|k|^{q}} \text {. }
\end{aligned}
$$

Thus,

$$
B\left(N_{1}, N_{2}, z\right) \leq\left(\frac{M_{E}}{\delta_{1}}\right)\left\{2 N_{E} 3^{q}+\sum_{\substack{k=-\infty \\ k \neq 0}}^{\infty} \frac{1}{|k|^{q}}\right\}^{1 / q} .
$$

In view of (39), it follows from (40), (41), and (45) that the series

$$
\sum_{\substack{k=-\infty \\ k \neq 0}}^{\infty} \frac{G_{\alpha}(\tau z)}{G_{\alpha}^{\prime}\left(j_{k}\right)\left(\tau z-j_{k}\right)} f\left(\frac{j_{k}}{\tau}\right)
$$

converges absolutely and uniformly on $E$, so its sum $L_{\tau, \alpha}(f ; z)$ defines an entire function.

Since $G_{\alpha}(\tau z)$ is an entire function of exponential type $\tau$, for each $\varepsilon>0$, there exists a constant $C=C(\varepsilon)$ depending on $\varepsilon$ such that

$$
\left|G_{\alpha}(\tau z)\right|<C e^{(\tau+\varepsilon)|z|} \quad z \in \mathbb{C} .
$$

By the definition of $N_{E}$, we have $\tau|z| \leq j_{N_{E}}$ for all $z \in E$, so if $E:=\{z \in \mathbb{C}:|z| \leq R\}$, then $\tau R \leq j_{N_{E}}$. Since $j_{k+1}-j_{k} \geq \delta_{1}$, there cannot be more than $\left[\frac{\tau R}{\delta_{1}}\right]$ positive zeros in $(0, \tau R]$, therefore, $N_{E} \leq \frac{\tau R}{\delta_{1}}$. From the above calculations, it follows that for $|z| \leq R$, $R>0$, we have

$$
\begin{aligned}
\left|L_{\tau, \alpha}(f ; z)\right| \leq \frac{1}{c_{2}}\left(\left.\left.\sum_{\substack{k=-\infty \\
k \neq 0}}^{\infty}|| j_{k}\right|^{\alpha+\frac{1}{2}} f\left(\frac{j_{k}}{\tau}\right)\right|^{p}\right)^{1 / p} & \\
& \times C \frac{e^{(\tau+\varepsilon)\left(R+\delta_{2}+\frac{\delta_{1}}{3 \tau}\right)}}{\delta_{1}}\left\{\left(\frac{2 \tau R}{\delta_{1}}\right) 3^{q}+\sum_{\substack{k=-\infty \\
k \neq 0}}^{\infty} \frac{1}{|k|^{q}}\right\}^{1 / q}
\end{aligned}
$$

where $\delta_{2}$ is as in (16). This implies that $L_{\tau, \alpha}(f ; \cdot)$ is of exponential type $\tau$. 
Lemma 13. Let $f$ be an entire function of exponential type $\tau>0$. If there exists $\delta>0$ such that $|x|^{\alpha+\frac{1}{2}} f(x) \in L^{p}(\mathbb{R} \backslash(-\delta, \delta))$ for some $\alpha>-1$ and some $p>1$, then

$$
f(z) \equiv L_{\tau, \alpha}(f ; z) \text {. }
$$

Remark 5. In particular, we can claim that if $f$ is an entire function of exponential type $\tau$ satisfying the conditions of Lemma 13, then it cannot vanish at all the zeros of $G_{\alpha}(\tau z)$ without being identically zero.

Proof. Consider the integral

$$
I_{N}(z):=\frac{1}{2 \pi i} \oint_{\mathcal{C}_{N}} \frac{f(\zeta)}{(z-\zeta) G_{\alpha}(\tau \zeta)} d \zeta
$$

where $\mathcal{C}_{N}$ is the circle $|\zeta|=R_{N}:=\left(N+\frac{\alpha}{2}+\frac{1}{4}\right) \frac{\pi}{\tau}$, and $N$ is a large positive integer. By the residue theorem,

$$
I_{N}(z)=-\frac{f(z)}{G_{\alpha}(\tau z)}+\sum_{\left|\frac{j_{k}}{\tau}\right|<R_{N}} \frac{f\left(\frac{j_{k}}{\tau}\right)}{\left(z-\frac{j_{k}}{\tau}\right) \tau G_{\alpha}^{\prime}\left(j_{k}\right)}
$$

if $|z|<R_{N}, z \neq j_{k} / \tau$. Hence, the desired result will follow if we show that as $N \rightarrow \infty$, $I_{N}(z) \rightarrow 0$ uniformly for all $z$ belonging to any compact set $E \subset \mathbb{C}$.

By Lemma 1, there exists a positive constant $c_{1}=c_{1}(\alpha)$ such that for all large $N$, say $N \geq N_{0}=N_{0}(\alpha)$, we have

$$
\left|I_{N}(z)\right| \leq \frac{1}{2 \pi c_{1}} \int_{-\pi}^{\pi} \frac{\left|\tau R_{N}\right|^{\alpha+\frac{1}{2}}\left|f\left(R_{N} e^{i \theta}\right)\right|}{e^{\tau R_{N}|\sin \theta|}} \frac{R_{N}}{\left|R_{N}-\right| z||} d \theta .
$$

By Lemma 4 , the assumption $|x|^{\alpha+\frac{1}{2}} f(x) \in L^{p}(\mathbb{R} \backslash(-\delta, \delta))$ implies that

$$
|x|^{\alpha+\frac{1}{2}} f(x) \longrightarrow 0 \quad \text { as } \quad x \rightarrow \pm \infty \text {. }
$$

Hence, there exists a constant $M_{1}$ such that

$$
|x|^{\alpha+\frac{1}{2}}|f(x)| \leq M_{1} \quad \text { for } \quad|x| \geq 1
$$

Let $M_{2}:=\max _{|z| \leq 1}|f(z)|, M:=\max \left\{M_{1}, M_{2}\right\}$. Consider the function

$$
\varphi(z):=\left(\tau\left(z+z_{0}\right)\right)^{\alpha+\frac{1}{2}} f(z) \text { where } z_{0}:=1+i
$$

Clearly, $\varphi$ is regular and of exponential type $\tau$ in the closed upper half-plane, and for $x \in \mathbb{R}$,

$$
\begin{aligned}
|\varphi(x)| & =\tau^{\alpha+\frac{1}{2}}\left((x+1)^{2}+1\right)^{\frac{1}{2}\left(\alpha+\frac{1}{2}\right)}|f(x)| \\
& \leq \begin{cases}(\sqrt{5} \tau)^{\alpha+\frac{1}{2}}|f(x)| & \text { if }|x| \leq 1 \\
(\sqrt{5} \tau)^{\alpha+\frac{1}{2}}|x|^{\alpha+\frac{1}{2}}|f(x)| & \text { if }|x| \geq 1\end{cases} \\
& \leq M_{0}
\end{aligned}
$$

where $M_{0}:=(\sqrt{5} \tau)^{\alpha+\frac{1}{2}} M$. By a well-known result [5, Theorem 6.2.4],

$$
|\varphi(x+i y)| \leq M_{0} e^{\tau y} \quad \text { for } \quad x \in \mathbb{R}, \quad 0 \leq y<\infty
$$

Now let

$$
g(z):=\varphi(z) e^{i \tau z}
$$

It is regular and of exponential type in the upper half-plane. Since $|g(x)|=|\varphi(x)| \leq$ $M_{0}$ for all $x \in \mathbb{R}$ and $h_{g}\left(\frac{\pi}{2}\right)=h_{\varphi}\left(\frac{\pi}{2}\right)-\tau \leq 0$, it follows [5, Theorem 6.2.4] that

$$
|g(x+i y)| \leq M \quad \text { for } \quad x \in \mathbb{R}, \quad 0 \leq y<\infty .
$$


By (47), we have

$$
\lim _{x \rightarrow \infty}|g(x)|=\lim _{x \rightarrow \infty}|\varphi(x)|=0 .
$$

Hence, the function $g$ satisfies the conditions of Lemma 9 with $a=0$, so

$$
\lim _{z \rightarrow \infty} g(z)=0
$$

uniformly in the sector $0 \leq \arg z \leq \frac{3 \pi}{4}$. Given $\varepsilon>0$, there exists $N_{1} \geq N_{0}(\alpha)$ such that for all $N>N_{1}$ and $\theta \in\left[0, \frac{3 \pi}{4}\right]$, we have $\left|g\left(R_{N} e^{i \theta}\right)\right|<\varepsilon$, i.e.,

$$
\frac{\left|\tau\left(R_{N} e^{i \theta}+1+i\right)\right|^{\alpha+\frac{1}{2}}\left|f\left(R_{N} e^{i \theta}\right)\right|}{e^{\tau R_{N} \sin \theta}}<\varepsilon .
$$

Thus,

$$
\frac{\left(\tau\left(R_{N}-\sqrt{2}\right)\right)^{\alpha+\frac{1}{2}}\left|f\left(R_{N} e^{i \theta}\right)\right|}{e^{\tau R_{N}|\sin \theta|}}<\varepsilon
$$

for all $N>N_{1}$ and $\theta \in\left[0, \frac{3 \pi}{4}\right]$. The same reasoning applied to the function $\varphi(-z)$ shows that (48) holds for all $\theta \in\left[\frac{\pi}{4}, \pi\right]$ as well, and therefore, for $\theta \in[0, \pi]$. Changing the value of $z_{0}$ to $1-i$ in the definition of $\varphi$ and applying the above considerations to the function $\overline{\varphi(\bar{z})}$, we conclude that (48) is true also for $\theta \in[-\pi, 0)$ and $N>N_{1}$. Hence, (48) is satisfied for $N>N_{1}$ without any restrictions on $\theta$.

Now let $E$ be an arbitrary compact subset of $\mathbb{C}$. For all sufficiently large $N$, say $N \geq N_{2} \geq N_{1}$

$$
\frac{R_{N}}{\left|R_{N}-\right| z||} \leq 2
$$

Using (48), (49) in (46), we see that as $N \rightarrow \infty,\left|I_{N}(z)\right| \rightarrow 0$ uniformly for all $z \in E$.

Remark 6. The results contained in [7], [11, Lemma 3], [17], [28], and [34] may be seen as being relevant to Lemma 13, but they do not contain it.

Lemma 14. Let $\alpha \geq-\frac{1}{2}, p>1$ or $-1<\alpha<-\frac{1}{2}, 1<p<\frac{2}{|1+2 \alpha|}$. If

$$
\sum_{\substack{k=-\infty \\ k \neq 0}}^{\infty}\left|\left(\frac{j_{k}}{\tau}\right)^{\alpha+\frac{1}{2}} f\left(\frac{j_{k}}{\tau}\right)\right|^{p}<\infty
$$

then there exists a constant $B_{\alpha, p}$ depending only on $\alpha$ and $p$ such that

$$
\int_{-\infty}^{\infty}\left|x^{\alpha+\frac{1}{2}} L_{\tau, \alpha}(f ; x)\right|^{p} d x \leq B_{\alpha, p}^{p} \frac{\pi}{\tau} \sum_{\substack{k=-\infty \\ k \neq 0}}^{\infty}\left|\frac{1}{\tau^{\alpha+\frac{1}{2}} G_{\alpha}^{\prime}\left(j_{k}\right)} f\left(\frac{j_{k}}{\tau}\right)\right|^{p} .
$$

Remark 7. This is an analogue of a classical inequality of J. Marcinkiewicz [19, Theorem 10] for trigonometric polynomials, and perhaps is the most nontrivial part of the paper.

Proof. Without loss of generality, we may assume $\tau=\pi$. Let

$$
f_{k}:=\frac{1}{G_{\alpha}^{\prime}\left(j_{k}\right)} f\left(\frac{j_{k}}{\pi}\right), \quad k= \pm 1, \pm 2, \ldots .
$$


Then, by (19),

$$
\begin{aligned}
\sum_{\substack{k=-\infty \\
k \neq 0}}^{\infty}\left|f_{k}\right|^{p} & \leq\left(\frac{1}{c_{2}}\right)^{p} \sum_{\substack{k=-\infty \\
k \neq 0}}^{\infty}\left|\left(j_{k}\right)^{\alpha+\frac{1}{2}} f\left(\frac{j_{k}}{\pi}\right)\right|^{p} \\
& =\left(\frac{\pi^{\alpha+\frac{1}{2}}}{c_{2}}\right)^{p} \sum_{\substack{k=-\infty \\
k \neq 0}}^{\infty}\left|\left(\frac{j_{k}}{\pi}\right)^{\alpha+\frac{1}{2}} f\left(\frac{j_{k}}{\pi}\right)\right|^{p} \\
& <\infty
\end{aligned}
$$

by (50), Hence, the right-hand side of (51) is finite.

Clearly $\int_{-\infty}^{\infty}\left|x^{\alpha+\frac{1}{2}} L_{\pi, \alpha}(f ; x)\right|^{p} d x=\sum_{n=-\infty}^{\infty} \mathcal{I}_{n}$ where

$$
\mathcal{I}_{n}:=\int_{n-\frac{1}{2}}^{n+\frac{1}{2}}\left|x^{\alpha+\frac{1}{2}} L_{\pi, \alpha}(f ; x)\right|^{p} d x, \quad n=0, \pm 1, \pm 2, \ldots .
$$

Denote by $k_{1}$ the smallest integer such that $j_{k} \geq 2$ for $k \geq k_{1}$. Then,

$$
\begin{aligned}
\mathcal{I}_{0} & =\int_{-\frac{1}{2}}^{\frac{1}{2}}\left|\sum_{k=-k_{1}+1}^{k_{1}-1} x^{\alpha+\frac{1}{2}} \frac{J_{\alpha}(\pi x)}{(\pi x)^{\alpha}\left(\pi x-j_{k}\right)} f_{k}+x^{\alpha+\frac{1}{2}} \frac{J_{\alpha}(\pi x)}{(\pi x)^{\alpha}} \sum_{|k| \geq k_{1}} \frac{1}{\pi x-j_{k}} f_{k}\right|^{p} d x \\
\leq & 2^{p-1}\left\{\int_{-\frac{1}{2}}^{\frac{1}{2}}\left|\sum_{\substack{k=-k_{1}+1 \\
k \neq 0}}^{k_{1}-1} x^{\alpha+\frac{1}{2}} \frac{J_{\alpha}(\pi x)}{(\pi x)^{\alpha}\left(\pi x-j_{k}\right)} f_{k}\right|^{p} d x\right. \\
& \left.+\left(\int_{-\frac{1}{2}}^{\frac{1}{2}}\left|x^{\alpha+\frac{1}{2}} \frac{J_{\alpha}(\pi x)}{(\pi x)^{\alpha}}\right|^{p} d x\right)\left(\sum_{|k| \geq k_{1}} \frac{1}{\left|j_{k}\right|-\frac{\pi}{2}}\left|f_{k}\right|\right)^{p}\right\} \\
\leq & 2^{p-1}\left\{\left(2 k_{1}-2\right)^{p-1} \sum_{k=-k_{1}+1}^{k_{1}-1}\left(\int_{-\frac{1}{2}}^{\frac{1}{2}}\left|x^{\alpha+\frac{1}{2}} \frac{J_{\alpha}(\pi x)}{(\pi x)^{\alpha}\left(\pi x-j_{k}\right)}\right|^{p} d x\right)\left|f_{k}\right|^{p}\right. \\
& \left.+\left(\int_{-\frac{1}{2}}^{\frac{1}{2}}\left|x^{\alpha+\frac{1}{2}} \frac{J_{\alpha}(\pi x)}{(\pi x)^{\alpha}}\right|^{p} d x\right)\left(\sum_{|k| \geq k_{1}}\left(\frac{1}{\left|j_{k}\right|-\frac{\pi}{2}}\right)^{\frac{p}{p-1}}\right)^{p-1} \sum_{|k| \geq k_{1}}\left|f_{k}\right|^{p}\right\} .
\end{aligned}
$$

If $\alpha \geq-\frac{1}{2}$, then for all $p>1$, whereas if $-1<\alpha<-\frac{1}{2}$, then for $1<p<\frac{2}{|1+2 \alpha|}$, there exists a constant $c_{3}=c_{3}(\alpha, p)$ such that

$$
\int_{-\frac{1}{2}}^{\frac{1}{2}}\left|x^{\alpha+\frac{1}{2}} \frac{J_{\alpha}(\pi x)}{(\pi x)^{\alpha}\left(\pi x-j_{k}\right)}\right|^{p} d x \leq c_{3} \quad \text { for } \quad k= \pm 1, \pm 2, \ldots, \pm\left(k_{1}-1\right) .
$$

The condition on $p$ also makes sure that

$$
\int_{-\frac{1}{2}}^{\frac{1}{2}}\left|x^{\alpha+\frac{1}{2}} \frac{J_{\alpha}(\pi x)}{(\pi x)^{\alpha}}\right|^{p} d x
$$

exists. Its value depends on $\alpha$ and $p$; we denote it by $c_{4}=c_{4}(\alpha, p)$. From (14), it follows that if $p>1$, then the series

$$
\sum_{|k| \geq k_{1}}\left(\frac{1}{\left|j_{k}\right|-\frac{\pi}{2}}\right)^{\frac{p}{p-1}}
$$


converges. Denote its sum by $c_{5}=c_{5}(\alpha, p)$. Thus

$$
\begin{aligned}
\mathcal{I}_{0} & \leq 2^{p-1}\left\{\left(2 k_{1}-2\right)^{p-1} c_{3} \sum_{\substack{k=-k_{1}+1 \\
k \neq 0}}^{k_{1}-1}\left|f_{k}\right|^{p}+c_{4} \cdot\left(c_{5}\right)^{p-1} \sum_{|k| \geq k_{1}}\left|f_{k}\right|^{p}\right\} \\
& \leq c_{6} \sum_{\substack{k=-\infty \\
k \neq 0}}^{\infty}\left|f_{k}\right|^{p}
\end{aligned}
$$

where

$$
c_{6}=c_{6}(\alpha, p):=\max \left\{2^{p-1}\left(2 k_{1}-2\right)^{p-1} c_{3}, 2^{p-1} c_{4} \cdot\left(c_{5}\right)^{p-1}\right\} .
$$

Now we proceed to estimate $\sum_{\substack{n=-\infty \\ n \neq 0}}^{\infty} \mathcal{I}_{n}$. Let

$$
\mathcal{L}_{n}:=\max _{n-\frac{1}{2} \leq x \leq n+\frac{1}{2}}\left|x^{\alpha+\frac{1}{2}} L_{\pi, \alpha}(f ; x)\right|, \quad n \neq 0 .
$$

Then, obviously

$$
\mathcal{I}_{n} \leq \mathcal{L}_{n}^{p}, \quad n \neq 0 .
$$

Let $\xi_{n}$ be a point in $\left[-\frac{1}{2}, \frac{1}{2}\right]$ such that

Then,

$$
\mathcal{L}_{n}=\left|\left(n+\xi_{n}\right)^{\alpha+\frac{1}{2}} L_{\pi, \alpha}\left(f ; n+\xi_{n}\right)\right| .
$$

$$
\mathcal{L}_{n}=\frac{1}{\pi^{\alpha+3 / 2}}\left|\sqrt{\pi\left(n+\xi_{n}\right)} J_{\alpha}\left(\pi\left(n+\xi_{n}\right)\right) \sum_{\substack{k=-\infty \\ k \neq 0}}^{\infty} \frac{f_{k}}{n+\xi_{n}-\frac{j_{k}}{\pi}}\right| .
$$

At this stage we need to obtain estimates for $\left|\sqrt{\pi x} J_{\alpha}(\pi x)\right|$ and $\left|\sqrt{\pi x} \frac{J_{\alpha}(\pi x)}{\pi x-j_{k}}\right|$. From the asymptotic formula (11), we have

$$
\frac{J_{\alpha}(z)}{z^{\alpha}} \sim \sqrt{\frac{2}{\pi}} \frac{1}{z^{\alpha+\frac{1}{2}}} \cos \left(z-\frac{\alpha \pi}{2}-\frac{\pi}{4}\right)+O\left(\frac{1}{z^{\alpha+\frac{3}{2}}}\right) \quad \text { for } \quad z \in \mathbb{R}, \quad z \rightarrow \infty .
$$

Consequently,

$$
\left|\frac{J_{\alpha}(z)}{z^{\alpha}}\right| \leq \frac{2}{\sqrt{\pi}} \frac{1}{|z|^{\alpha+\frac{1}{2}}} \quad \text { for } \quad z \in \mathbb{R}, \quad z \rightarrow \pm \infty .
$$

The function $\frac{J_{\alpha}(z)}{z^{\alpha}}$ is entire, so, if $d:=\min \left\{\pi, j_{1}\right\}$, there exists a constant $c_{7}=c_{7}(\alpha)$ such that

$$
|x|^{\alpha+\frac{1}{2}}\left|\frac{J_{\alpha}(x)}{x^{\alpha}}\right| \leq c_{7} \quad \text { for } \quad x \in \mathbb{R} \backslash\left[-\frac{d}{2}, \frac{d}{2}\right] .
$$

Thus,

$$
\left|\sqrt{\pi x} J_{\alpha}(\pi x)\right| \leq c_{7} \quad \text { for } \quad x \in \mathbb{R} \backslash\left[-\frac{d}{2 \pi}, \frac{d}{2 \pi}\right]
$$

From (15), we have

$$
j_{k+1}-j_{k} \geq 2 \pi \delta^{\prime}, \quad \delta^{\prime}=\frac{\delta_{1}}{2 \pi} .
$$

Since $\sqrt{\pi z} J_{\alpha}(\pi z)$ is holomorphic and of exponential type in $\mathfrak{R e} z \geq \frac{d}{2 \pi}$, it follows from (56) that for $z \in \Delta_{\alpha}:=\left\{z=x+i y: x \geq \frac{d}{2 \pi},|y| \leq \delta^{\prime}\right\}$, we have [5, Theorem 6.2.4]

$$
\left|\sqrt{\pi z} J_{\alpha}(\pi z)\right| \leq c_{8}
$$


where $c_{8}=c_{8}(\alpha)$ is a constant. For each $k \in \mathbb{N}$, let $D_{k}\left(\delta^{\prime}\right):=\left\{z:\left|z-\frac{j_{k}}{\pi}\right|<\delta^{\prime}\right\}$. Then for all $z$ in $\Delta_{\alpha} \backslash D_{k}\left(\delta^{\prime}\right)$, we have

$$
\left|\sqrt{\pi z} \frac{J_{\alpha}(\pi z)}{\pi z-j_{k}}\right| \leq \frac{c_{8}}{\pi \delta}=: c_{9}(\alpha)=c_{9} .
$$

In particular, (58) holds for $z$ belonging to the boundary of the disk $D_{k}\left(\delta^{\prime}\right)$ on and inside which the function $\sqrt{\pi z} \frac{J_{\alpha}(\pi z)}{\pi z-j_{k}}$ is holomorphic. Hence, by the maximum modulus principle, (58) holds also for $z \in D_{k}\left(\delta^{\prime}\right)$, i.e., it is true for all $z \in \Delta_{\alpha}$.

Amongst the points $\left\{\frac{j_{k}}{\pi}\right\}_{k \in \mathbb{Z}}$, there are possibly two which are closest to $n+\xi_{n}$. Let $\frac{j_{k_{n}}}{\pi}$ be (any) one of them. In view of (57), we have

$$
\left|n+\xi_{n}-\frac{j_{k}}{\pi}\right| \geq \delta^{\prime} \quad \text { for } \quad k \in \mathbb{Z} \backslash\left\{k_{n}\right\} .
$$

Now, let us return to (55) and write

$$
\frac{1}{n+\xi_{n}-\frac{j_{k}}{\pi}}=\frac{1}{n-k+\frac{1}{2}}+\frac{\frac{j_{k}}{\pi}-k-\xi_{n}+\frac{1}{2}}{\left(n+\xi_{n}-\frac{j_{k}}{\pi}\right)\left(n-k+\frac{1}{2}\right)} .
$$

So, by (56)

$$
\begin{aligned}
\mathcal{L}_{n} \leq & \frac{c_{7}}{\pi^{\alpha+3 / 2}}\left|\sum_{\substack{k=-\infty \\
k \neq 0}}^{\infty} \frac{f_{k}}{n-k+\frac{1}{2}}\right| \\
& +\left|\sum_{\substack{k=-\infty \\
k \neq 0}}^{\infty} \frac{1}{\pi^{\alpha+\frac{1}{2}}} \cdot \frac{\sqrt{\pi\left(n+\xi_{n}\right)} J_{\alpha}\left(\pi\left(n+\xi_{n}\right)\right)}{\pi\left(n+\xi_{n}\right)-j_{k}} \cdot \frac{\frac{j_{k}}{\pi}-k-\xi_{n}+\frac{1}{2}}{n-k+\frac{1}{2}} f_{k}\right| .
\end{aligned}
$$

Using, in the second sum on the right, the estimate (58) for $k=k_{n}$ and (56) for all other $k$, we obtain

$$
\begin{aligned}
\mathcal{L}_{n} \leq & \frac{c_{7}}{\pi^{\alpha+3 / 2}}\left|\sum_{\substack{k=-\infty \\
k \neq 0}}^{\infty} \frac{f_{k}}{n-k+\frac{1}{2}}\right|+\frac{c_{9}}{\pi^{\alpha+\frac{1}{2}}}\left|\frac{\frac{j_{k_{n}}}{\pi}-k_{n}-\xi_{n}+\frac{1}{2}}{n-k_{n}+\frac{1}{2}}\right|\left|f_{k_{n}}\right| \\
& +\frac{c_{7}}{\pi^{\alpha+3 / 2}} \sum_{\substack{k=-\infty \\
k \neq 0, k_{n}}}^{\infty} \frac{\left|\frac{j_{k}}{\pi}-k-\xi_{n}+\frac{1}{2}\right|}{\left|n+\xi_{n}-\frac{j_{k}}{\pi}\right| \cdot\left|n-k+\frac{1}{2}\right|}\left|f_{k}\right| .
\end{aligned}
$$

From (16), it follows that $\left|n+\xi_{n}-\frac{j_{k_{n}}}{\pi}\right| \leq \delta^{\prime \prime}:=\frac{\delta_{2}}{2 \pi}$ and

$$
\frac{\left|\frac{j_{k_{n}}}{\pi}-k_{n}-\xi_{n}+\frac{1}{2}\right|}{\left|n-k_{n}+\frac{1}{2}\right|} \leq 1+\frac{\left|n+\xi_{n}-\frac{j_{k_{n}}}{\pi}\right|}{\left|n-k_{n}+\frac{1}{2}\right|} \leq 1+2 \delta^{\prime \prime},
$$

since $n, k_{n} \in \mathbb{Z}$. Again, from (14), it follows that if $\beta_{k}:=j_{k} / \pi-k$, then

$$
\left|\beta_{k}\right| \leq c_{10}
$$

for some positive number $c_{10}=c_{10}(\alpha)$ and

$$
\left|\frac{j_{k}}{\pi}-k-\xi_{n}+\frac{1}{2}\right| \leq c_{10}+1 .
$$

Using the last three inequalities in (60), we obtain

$$
\mathcal{L}_{n} \leq\left|b_{n}\right|+\left|v_{n}\right|
$$


where

$$
\begin{gathered}
b_{n}:=\frac{c_{7}}{\pi^{\alpha+3 / 2}} \sum_{\substack{k=-\infty \\
k \neq 0}}^{\infty} \frac{f_{k}}{n-k+\frac{1}{2}}, \\
v_{n}:=\frac{c_{9}}{\pi^{\alpha+\frac{1}{2}}}\left(1+2 \delta^{\prime \prime}\right)\left|f_{k_{n}}\right|+\frac{c_{7}\left(c_{10}+1\right)}{\pi^{\alpha+3 / 2}} \sum_{\substack{k=-\infty \\
k \neq 0, k_{n}}}^{\infty} \frac{1}{\left|n-k-\beta_{k}+\xi_{n}\right| \cdot\left|n-k+\frac{1}{2}\right|}\left|f_{k}\right| .
\end{gathered}
$$

Defining $a_{0}=0, a_{k}:=\left(c_{7} / \pi^{\alpha+3 / 2}\right) f_{-k}$ for $k \neq 0$, we write $b_{n}$ in the form $\frac{1}{\pi} \sum_{k=-\infty}^{\infty} \frac{a_{k}}{k+n+\frac{1}{2}}$ where, in view of (52), $\sum_{k=-\infty}^{\infty}\left|a_{k}\right|^{p}$ converges. Lemma 10 implies that

$$
\sum_{\substack{k=-\infty \\ k \neq 0}}^{\infty}\left|b_{n}\right|^{p} \leq N_{p}^{p}\left(\frac{c_{7}}{\pi^{\alpha+3 / 2}}\right)^{p} \sum_{\substack{k=-\infty \\ k \neq 0}}^{\infty}\left|f_{k}\right|^{p}
$$

where $N_{p}$ is a constant depending only on $p$. We write $v_{n}$ in the form $\sum_{k=-\infty}^{\infty} t_{n-k}\left|u_{k}\right|$ by putting

$$
\begin{gathered}
u_{0}=0, \quad u_{k}=f_{k} \text { for } k \neq 0 ; \quad t_{n-k_{n}}=\frac{c_{9}\left(1+2 \delta^{\prime \prime}\right)}{\pi^{\alpha+\frac{1}{2}}}, \quad t_{n}=1, \\
t_{\mu}:=\frac{c_{7}\left(c_{10}+1\right)}{\pi^{\alpha+3 / 2}} \cdot \frac{1}{\left|\mu-\beta_{n-\mu}+\xi_{n}\right|\left|\mu+\frac{1}{2}\right|} \quad \text { for } \quad \mu \neq n, n-k_{n} .
\end{gathered}
$$

Referring to (52), we see that $\sum_{k=-\infty}^{\infty}\left|u_{k}\right|^{p}<\infty$. Further, using (59) and (62), we conclude that $t:=\sum_{\mu=-\infty}^{\infty} t_{\mu}<\infty$, so Lemma 11 may be applied to obtain

$$
\sum_{\substack{n=-\infty \\ n \neq 0}}^{\infty}\left|v_{n}\right|^{p} \leq t^{p} \sum_{\substack{k=-\infty \\ k \neq 0}}^{\infty}\left|f_{k}\right|^{p} .
$$

By (64), we have

$$
\sum_{\substack{n=-\infty \\ n \neq 0}}^{\infty} \mathcal{L}_{n}^{p} \leq 2^{p}\left(\sum_{\substack{n=-\infty \\ n \neq 0}}^{\infty}\left|b_{n}\right|^{p}+\sum_{\substack{n=-\infty \\ n \neq 0}}^{\infty}\left|v_{n}\right|^{p}\right) .
$$

Combined with (54), this gives

$$
\begin{aligned}
\sum_{\substack{n=-\infty \\
n \neq 0}}^{\infty} \mathcal{I}_{n} & \leq 2^{p}\left(\sum_{\substack{n=-\infty \\
n \neq 0}}^{\infty}\left|b_{n}\right|^{p}+\sum_{\substack{n=-\infty \\
n \neq 0}}^{\infty}\left|v_{n}\right|^{p}\right) \\
& \leq 2^{p}\left(N_{p}^{p}\left(\frac{c_{7}}{\pi^{\alpha+3 / 2}}\right)^{p}+t^{p}\right) \sum_{\substack{k=-\infty \\
k \neq 0}}^{\infty}\left|f_{k}\right|^{p}
\end{aligned}
$$

by (65) and (66). Now recalling the bound for $\mathcal{I}_{0}$ from (53), we get

$$
\begin{aligned}
\int_{-\infty}^{\infty}\left|x^{\alpha+\frac{1}{2}} L_{\pi, \alpha}(f ; x)\right|^{p} d x & =\sum_{n=-\infty}^{\infty} \mathcal{I}_{n} \\
& \leq\left(c_{6}+2^{p}\left(N_{p}^{p}\left(\frac{c_{7}}{\pi^{\alpha+3 / 2}}\right)^{p}+t^{p}\right)\right) \sum_{\substack{k=-\infty \\
k \neq 0}}^{\infty}\left|f_{k}\right|^{p}
\end{aligned}
$$


which is equivalent to (51).

\subsubsection{Approximation by step functions.}

Lemma 15. (i) Let $|x|^{\alpha+\frac{1}{2}} f(x) \in L^{p}(\mathbb{R})$ for some $\alpha \geq-\frac{1}{2}$ and some $p>1$. If $f \in L^{p}(-1,1)$, then for every $\varepsilon>0$, there exists a step function $\Omega$ with compact support such that

$$
\left(\int_{-1}^{1}|f(x)-\Omega(x)|^{p} d x\right)^{1 / p}<\varepsilon, \quad\left(\int_{|x| \geq 1}\left|x^{\alpha+\frac{1}{2}}(f(x)-\Omega(x))\right|^{p} d x\right)^{1 / p}<\varepsilon .
$$

(ii) Let $-1<\alpha<-\frac{1}{2}, 1<p<\frac{2}{|1+2 \alpha|}$. If $f \in \mathcal{F}^{\alpha, p}(\delta)$ for some $\delta>0$, then for every $\varepsilon>0$, there exists a step function $\Omega$ with compact support such that

$$
\begin{array}{r}
\left(\int_{-1}^{1}\left|x^{\alpha+\frac{1}{2}}(f(x)-\Omega(x))\right|^{p} d x\right)^{1 / p}<\varepsilon, \\
\left(\int_{|x| \geq 1}\left|x^{\alpha+\frac{1}{2}}(f(x)-\Omega(x))\right|^{p} d x\right)^{1 / p}<\varepsilon, \\
\max _{u \in \mathbb{R}}\left(\int_{-3}^{3}\left|x^{\alpha+\frac{1}{2}}(f(x-u)-\Omega(x-u))\right|^{p} d x\right)^{1 / p}<\varepsilon .
\end{array}
$$

Proof. (i) Since $f \in L^{p}(-1,1)$, there exists a step function $\Omega_{1}$ vanishing outside $(-1,1)$ such that [29, p. 118, problem 14]

$$
\left(\int_{-1}^{1}\left|f(x)-\Omega_{1}(x)\right|^{p} d x\right)^{1 / p}<\varepsilon
$$

The assumption $|x|^{\alpha+\frac{1}{2}} f(x) \in L^{p}(\mathbb{R})$ implies that for some $Y_{0}>1$ and all $Y \geq Y_{0}$,

$$
\int_{Y}^{\infty}\left|x^{\alpha+\frac{1}{2}} f(x)\right|^{p} d x<\frac{\varepsilon^{p}}{4}, \quad \int_{-\infty}^{-Y}\left|x^{\alpha+\frac{1}{2}} f(x)\right|^{p} d x<\frac{\varepsilon^{p}}{4} .
$$

Clearly, $f \in L^{p}\left(1, Y_{0}\right)$ and $f \in L^{p}\left(-Y_{0},-1\right)$. Hence, there exists a step function $\Omega_{2}$ vanishing outside $\left[-Y_{0},-1\right] \cup\left[1, Y_{0}\right]$ such that

$$
\begin{aligned}
& \int_{1}^{Y_{0}}\left|f(x)-\Omega_{2}(x)\right|^{p} d x<\frac{\varepsilon^{p}}{4 Y_{0}^{p\left(\alpha+\frac{1}{2}\right)}} \\
& \int_{-Y_{0}}^{-1}\left|f(x)-\Omega_{2}(x)\right|^{p} d x<\frac{\varepsilon^{p}}{4 Y_{0}^{p\left(\alpha+\frac{1}{2}\right)}} .
\end{aligned}
$$

Thus, if

$$
\Omega(x):= \begin{cases}\Omega_{1}(x) & \text { for } x \in(-1,1), \\ 0 & \text { for } x \in\left(-\infty,-Y_{0}\right) \cup\left(Y_{0}, \infty\right), \\ \Omega_{2}(x) & \text { for } x \in\left[-Y_{0},-1\right] \cup\left[1, Y_{0}\right],\end{cases}
$$


then clearly

$$
\begin{gathered}
\left(\int_{-1}^{1}|f(x)-\Omega(x)|^{p} d x\right)^{1 / p}<\varepsilon \\
\int_{|x| \geq 1}\left|x^{\alpha+\frac{1}{2}}(f(x)-\Omega(x))\right|^{p} d x=\int_{-\infty}^{-Y_{0}}\left|x^{\alpha+\frac{1}{2}} f(x)\right|^{p} d x+\int_{Y_{0}}^{\infty}\left|x^{\alpha+\frac{1}{2}} f(x)\right|^{p} d x \\
+\int_{-Y_{0}}^{-1}\left|x^{\alpha+\frac{1}{2}}(f(x)-\Omega(x))\right|^{p} d x+\int_{1}^{Y_{0}}\left|x^{\alpha+\frac{1}{2}}(f(x)-\Omega(x))\right|^{p} d x \\
<\frac{\varepsilon^{p}}{4}+\frac{\varepsilon^{p}}{4}+\frac{\varepsilon^{p}}{4}+\frac{\varepsilon^{p}}{4}
\end{gathered}
$$

i.e.,

$$
\left(\int_{|x| \geq 1}\left|x^{\alpha+\frac{1}{2}}(f(x)-\Omega(x))\right|^{p} d x\right)^{1 / p}<\varepsilon .
$$

(ii) Let $\beta_{p}:=\frac{\left|p\left(\alpha+\frac{1}{2}\right)\right|+1}{|p(2 \alpha+1)|}$ and note that $\beta_{p}>1,\left|p\left(\alpha+\frac{1}{2}\right)\right| \beta_{p}<1$. Further, let

$$
\gamma_{\alpha, p}:=\left(\int_{-3}^{3} s^{p\left(\alpha+\frac{1}{2}\right) \beta_{p}} d s\right)^{1 / \beta_{p}}
$$

and set

$$
\varepsilon_{1}:=\min \left\{\left(\frac{\varepsilon}{\left(\gamma_{\alpha, p}\right)^{1 / p} M^{1 / \beta_{p}}}\right)^{\frac{\beta_{p}}{\beta_{p}-1}}, \varepsilon\right\}
$$

where $M:=\sup _{x \in \mathbb{R}}|f(x)|<\infty$.

There exists a $Y_{0}>1, \delta_{0} \in(0,1)$ such that

$$
\begin{gathered}
\int_{-\infty}^{-Y_{0}}\left|x^{\alpha+\frac{1}{2}} f(x)\right|^{p} d x<\frac{\varepsilon_{1}^{p}}{5}, \quad \int_{Y_{0}}^{\infty}\left|x^{\alpha+\frac{1}{2}} f(x)\right|^{p} d x<\frac{\varepsilon_{1}^{p}}{5}, \\
\int_{Y-3}^{Y+3}|f(x)|^{p} d x<\frac{\varepsilon_{1}^{p}}{5} \quad \text { for }|Y|>Y_{0} \\
\int_{-\delta_{0}}^{\delta_{0}}|f(x)|^{p} d x \leq \int_{-\delta_{0}}^{\delta_{0}}\left|x^{\alpha+\frac{1}{2}} f(x)\right|^{p} d x<\frac{\varepsilon_{1}^{p}}{5} .
\end{gathered}
$$

Clearly, $f$ belongs to $L^{p}\left(-Y_{0},-1\right)$ and to $L^{p}\left(1, Y_{0}\right)$. Hence, there exists a step function $\Omega_{1}$ vanishing outside $\left[-Y_{0},-1\right] \cup\left[1, Y_{0}\right]$ such that

$$
\int_{-Y_{0}}^{-1}\left|f(x)-\Omega_{1}(x)\right|^{p} d x<\frac{\varepsilon_{1}^{p}}{5}, \quad \int_{1}^{Y_{0}}\left|f(x)-\Omega_{1}(x)\right|^{p} d x<\frac{\varepsilon_{1}^{p}}{5}
$$

so a fortiori

$$
\int_{-Y_{0}}^{-1}\left|x^{\alpha+\frac{1}{2}}\left(f(x)-\Omega_{1}(x)\right)\right|^{p} d x<\frac{\varepsilon_{1}^{p}}{5}, \quad \int_{1}^{Y_{0}}\left|x^{\alpha+\frac{1}{2}}\left(f(x)-\Omega_{1}(x)\right)\right|^{p} d x<\frac{\varepsilon_{1}^{p}}{5} .
$$

Since $f$ belongs to $L^{p}\left(-1,-\delta_{0}\right)$ and to $L^{p}\left(\delta_{0}, 1\right)$, there exists a step function $\Omega_{2}$ vanishing outside $\left(-1,-\delta_{0}\right] \cup\left[\delta_{0}, 1\right)$ such that

$$
\int_{-1}^{-\delta_{0}}\left|f(x)-\Omega_{2}(x)\right|^{p} d x<\frac{\varepsilon_{1}^{p}}{5} \delta_{0}^{p\left|\alpha+\frac{1}{2}\right|}, \quad \int_{\delta_{0}}^{1}\left|f(x)-\Omega_{2}(x)\right|^{p} d x<\frac{\varepsilon_{1}^{p}}{5} \delta_{0}^{p\left|\alpha+\frac{1}{2}\right|} .
$$


Consequently,

$$
\int_{-1}^{-\delta_{0}}\left|x^{\alpha+\frac{1}{2}}\left(f(x)-\Omega_{2}(x)\right)\right|^{p} d x<\frac{\varepsilon_{1}^{p}}{5}, \quad \int_{\delta_{0}}^{1}\left|x^{\alpha+\frac{1}{2}}\left(f(x)-\Omega_{2}(x)\right)\right|^{p} d x<\frac{\varepsilon_{1}^{p}}{5} .
$$

The step functions $\Omega_{1}, \Omega_{2}$ may be chosen such that $0 \leq \Omega_{j}(x) \leq M, j=1,2$, at points where $f(x) \geq 0$, and $-M \leq \Omega_{j}(x) \leq 0$ when $f(x) \leq 0$. Now put

$$
\Omega(x):= \begin{cases}0 & \text { for }|x|>Y_{0} \text { or }|x|<\delta_{0}, \\ \Omega_{1}(x) & \text { for } 1 \leq|x| \leq Y_{0} \\ \Omega_{2}(x) & \text { for } \delta_{0} \leq|x|<1\end{cases}
$$

From (72) and (76), it follows that

$$
\left(\int_{-1}^{1}\left|x^{\alpha+\frac{1}{2}}(f(x)-\Omega(x))\right|^{p} d x\right)^{1 / p}<\left(\frac{3}{5}\right)^{1 / p} \varepsilon_{1}<\varepsilon
$$

whereas (70) and (74) imply

$$
\left(\int_{|x| \geq 1}\left|x^{\alpha+\frac{1}{2}}(f(x)-\Omega(x))\right|^{p} d x\right)^{1 / p}<\varepsilon .
$$

For all $u \in \mathbb{R}$,

$$
\begin{aligned}
& \int_{-3}^{3} \mid x^{\alpha+\frac{1}{2}}f(x-u)-\Omega(x-u))\left.\right|^{p} d x \\
&=\int_{-u-3}^{-u+3}|u+t|^{p\left(\alpha+\frac{1}{2}\right)}|f(t)-\Omega(t)|^{p} d t \\
& \leq\left(\int_{-u-3}^{-u+3}|u+t|^{p\left(\alpha+\frac{1}{2}\right) \beta_{p}} d t\right)^{1 / \beta_{p}} \cdot\left(\int_{-u-3}^{-u+3}|f(t)-\Omega(t)|^{\frac{p \beta_{p}}{\beta_{p}-1}} d t\right)^{\frac{\beta_{p}-1}{\beta_{p}}} \\
& \leq \gamma_{\alpha, p} \cdot \max _{u \in \mathbb{R}}\left(\int_{u-3}^{u+3}|f(t)-\Omega(t)|^{\frac{p \beta_{p}}{\beta_{p}-1}} d t\right)^{\frac{\beta_{p}-1}{\beta_{p}}} .
\end{aligned}
$$

Since $|f(t)-\Omega(t)| \leq M$ for all $t$, we have

$$
\int_{u-3}^{u+3}|f(t)-\Omega(t)|^{\frac{p \beta_{p}}{\beta_{p}-1}} d t \leq M^{\frac{p}{\beta_{p}-1}} \int_{u-3}^{u+3}|f(t)-\Omega(t)|^{p} d t<M^{\frac{p}{\beta_{p}-1}} \varepsilon_{1}^{p}
$$

by. (71)-(73) and (75). Hence,

$$
\max _{u \in \mathbb{R}}\left(\int_{-3}^{3}\left|x^{\alpha+\frac{1}{2}}(f(x-u)-\Omega(x-u))\right|^{p} d x\right)^{1 / p}<\gamma_{\alpha, p}^{\frac{1}{p}} M^{\frac{1}{\beta_{p}}} \varepsilon_{1}^{\frac{\beta_{p}-1}{\beta_{p}}} \leq \varepsilon .
$$

3.1.3. An auxiliary function and its properties. Given $f: \mathbb{R} \rightarrow \mathbb{C}$ and $\alpha>-1$, we define for each $\tau>0$ the function

$$
S_{\tau, \alpha}(f ; z):=\frac{\tau}{A} \int_{-\infty}^{\infty} f(t) \frac{G_{\alpha}(\tau(z-t))}{(\tau(z-t))^{2}-j_{1}^{2}} d t
$$

where $A:=\int_{-\infty}^{\infty} G_{\alpha}(t) /\left(t^{2}-j_{1}^{2}\right) d t ; A \neq 0$ by Lemma 3 .

For real $z$, we also may write

$$
S_{\tau, \alpha}(f ; z)=\frac{\tau}{A} \int_{-\infty}^{\infty} f(z+t) \frac{G_{\alpha}(\tau t)}{(\tau t)^{2}-j_{1}^{2}} d t
$$


Lemmas 16-19 contain facts about $S_{\tau, \alpha}(f ; \cdot)$ which we need for the proof of our theorem on the mean convergence of $L_{\tau, \alpha}(f ; \cdot)$.

Lemma 16. (i) Given $\alpha \geq-\frac{1}{2}, p>1$, let $|x|^{\alpha+\frac{1}{2}} f(x) \in L^{p}(\mathbb{R})$ and $f \in L^{p}(-1,1)$. Then, for $\tau \geq 1$

$$
\begin{aligned}
\left\|S_{\tau, \alpha}(f ; \cdot)\right\|_{\alpha, p} & :=\left(\int_{-\infty}^{\infty}\left|x^{\alpha+\frac{1}{2}} S_{\tau, \alpha}(f ; x)\right|^{p} d x\right)^{1 / p} \\
& \leq C_{\alpha, p}\left\{\left(\int_{-1}^{1}|f(x)|^{p} d x\right)^{1 / p}+\left(\int_{|x| \geq 1}\left|x^{\alpha+\frac{1}{2}} f(x)\right|^{p} d x\right)^{1 / p}\right\}
\end{aligned}
$$

where $C_{\alpha, p}$ is a constant depending only on $\alpha$ and $p$.

(ii) If $-1<\alpha<-\frac{1}{2}, 1<p<\frac{2}{|2 \alpha+1|}$, and $f \in \mathcal{F}^{\alpha, p}(\delta)$ for some $\delta>0$, then

$$
\begin{aligned}
& \left\|S_{\tau, \alpha}(f ; \cdot)\right\|_{\alpha, p} \leq C_{\alpha, p}^{\prime}\left\{\left(\int_{-1}^{1}\left|x^{\alpha+\frac{1}{2}} f(x)\right|^{p} d x\right)^{1 / p}\right. \\
+ & \left.\left(\int_{|x| \geq 1}\left|x^{\alpha+\frac{1}{2}} f(x)\right|^{p} d x\right)^{1 / p}+\max _{u \in \mathbb{R}}\left(\int_{-3}^{3}\left|x^{\alpha+\frac{1}{2}} f(x-u)\right|^{p} d x\right)^{1 / p}\right\}
\end{aligned}
$$

where $C_{\alpha, p}^{\prime}$ is a constant depending only on $\alpha$ and $p$.

Remark 8. The estimate in (78) holds if $|x|^{\alpha+\frac{1}{2}} f(x) \in L^{p}(\mathbb{R})$ except that

$$
\max _{u \in \mathbb{R}}\left(\int_{-3}^{3}\left|x^{\alpha+\frac{1}{2}} f(x-u)\right|^{p} d x\right)^{1 / p}
$$

may possibly be $+\infty$.

Proof. (i) Since $|x|^{\alpha+\frac{1}{2}} f(x) \in L^{p}(\mathbb{R})$ and $f \in L^{p}(-1,1)$, the function $f$ must belong to $L^{p}(\mathbb{R})$. Set $B(z):=G_{\alpha}(z) /\left(z^{2}-j_{1}^{2}\right)$. Then

$$
\begin{aligned}
\left\|S_{\tau, \alpha}(f ; \cdot)\right\|_{\alpha, p} & =\frac{\tau}{A}\left(\int_{-\infty}^{\infty}\left|x^{\alpha+\frac{1}{2}} \int_{-\infty}^{\infty} f(t) B(\tau(x-t)) d t\right|^{p} d x\right)^{1 / p} \\
& =\frac{\tau}{A}\left(\int_{-\infty}^{\infty}\left|x^{\alpha+\frac{1}{2}} \int_{-\infty}^{\infty} f(x-t) B(\tau t) d t\right|^{p} d x\right)^{1 / p} \\
& \leq \frac{\tau}{A} \int_{-\infty}^{\infty}|B(\tau t)|\left(\int_{-\infty}^{\infty}\left|x^{\alpha+\frac{1}{2}} f(x-t)\right|^{p} d x\right)^{1 / p} d t
\end{aligned}
$$

by the generalized Minkowski inequality [24, p. 21, Section 1.3.2]. Now, using the fact that

$$
(|a|+|b|)^{\lambda} \leq \begin{cases}|a|^{\lambda}+|b|^{\lambda} & \text { if } 0<\lambda \leq 1 \\ 2^{\lambda-1}\left(|a|^{\lambda}+|b|^{\lambda}\right) & \text { if } 1 \leq \lambda<\infty\end{cases}
$$


we obtain

$$
\begin{gathered}
\left\|S_{\tau, \alpha}(f ; \cdot)\right\|_{\alpha, p} \leq \frac{\tau}{A} \int_{-\infty}^{\infty}|B(\tau t)|\left(\int_{-\infty}^{\infty}\left|(x+t)^{\alpha+\frac{1}{2}} f(x)\right|^{p} d x\right)^{1 / p} d t \\
\leq \frac{\tau}{A} \max \left\{1,2^{\alpha-\frac{1}{2}}\right\} \int_{-\infty}^{\infty}|B(\tau t)|\left\{\int_{-\infty}^{\infty}\left(\left(|x|^{\alpha+\frac{1}{2}}+|t|^{\alpha+\frac{1}{2}}\right)|f(x)|\right)^{p} d x\right\}^{1 / p} d t \\
\leq \frac{\tau}{A} \max \left\{1,2^{\alpha-\frac{1}{2}}\right\} 2^{1-\frac{1}{p}} \int_{-\infty}^{\infty}|B(\tau t)|\left(\int_{-\infty}^{\infty}\left|x^{\alpha+\frac{1}{2}} f(x)\right|^{p} d x\right. \\
\left.+|t|^{p\left(\alpha+\frac{1}{2}\right)} \int_{-\infty}^{\infty}|f(x)|^{p} d x\right)^{1 / p} d t \\
\leq \frac{\tau}{A} \max \left\{1,2^{\alpha-\frac{1}{2}}\right\} 2^{1-\frac{1}{p}} \int_{-\infty}^{\infty}|B(\tau t)|\left\{\left(\int_{-\infty}^{\infty}\left|x^{\alpha+\frac{1}{2}} f(x)\right|^{p} d x\right)^{1 / p}\right. \\
\left.+|t|^{\alpha+\frac{1}{2}}\left(\int_{-\infty}^{\infty}|f(x)|^{p} d x\right)^{1 / p}\right\} d t
\end{gathered}
$$

If $\tau \geq 1$, then

$$
\int_{-\infty}^{\infty} \tau|B(\tau t)| \cdot|t|^{\alpha+\frac{1}{2}} d t=\int_{-\infty}^{\infty}|B(u)|\left|\frac{u}{\tau}\right|^{\alpha+\frac{1}{2}} d u \leq \int_{-\infty}^{\infty}\left|u^{\alpha+\frac{1}{2}} B(u)\right| d u,
$$

so

$$
\begin{aligned}
\left\|S_{\tau, \alpha}(f ; \cdot)\right\|_{\alpha, p} \leq \frac{1}{A} \max & \left\{1,2^{\alpha-\frac{1}{2}}\right\} \cdot 2^{1-\frac{1}{p}} \max \left\{\int_{-\infty}^{\infty}|B(u)| d u, \int_{-\infty}^{\infty}\left|u^{\alpha+\frac{1}{2}} B(u)\right| d u\right\} \\
& \times\left\{\left(\int_{-\infty}^{\infty}\left|x^{\alpha+\frac{1}{2}} f(x)\right|^{p} d x\right)^{1 / p}+\left(\int_{-\infty}^{\infty}|f(x)|^{p} d x\right)^{1 / p}\right\} .
\end{aligned}
$$

Clearly,

$$
\begin{gathered}
\int_{-\infty}^{\infty}|f(x)|^{p} d x \leq \int_{-1}^{1}|f(x)|^{p} d x+\int_{|x| \geq 1}\left|x^{\alpha+\frac{1}{2}} f(x)\right|^{p} d x \\
\int_{-\infty}^{\infty}\left|x^{\alpha+\frac{1}{2}} f(x)\right|^{p} d x \leq \int_{-1}^{1}|f(x)|^{p} d x+\int_{|x| \geq 1}\left|x^{\alpha+\frac{1}{2}} f(x)\right|^{p} d x
\end{gathered}
$$

so

$$
\begin{gathered}
\left\|S_{\tau, \alpha}(f ; \cdot)\right\|_{\alpha, p} \leq \frac{2}{A} \max \left\{1,2^{\alpha-\frac{1}{2}}\right\} \cdot 2^{1-\frac{1}{p}} \max \left\{\int_{-\infty}^{\infty}|B(u)| d u, \int_{-\infty}^{\infty}\left|u^{\alpha+\frac{1}{2}} B(u)\right| d u\right\} \\
\times\left\{\left(\int_{|x| \geq 1}\left|x^{\alpha+\frac{1}{2}} f(x)\right|^{p} d x\right)^{1 / p}+\left(\int_{-1}^{1}|f(x)|^{p} d x\right)^{1 / p}\right\}
\end{gathered}
$$

i.e., (77) holds.

(ii) From (79), we have

$$
\left\|S_{\tau, \alpha}(f ; \cdot)\right\|_{\alpha, p} \leq \frac{\tau}{A}\left(\int_{-1}^{1}+\int_{|t| \geq 1}\right)|B(\tau t)|\left(\int_{-\infty}^{\infty}\left|(x+t)^{\alpha+\frac{1}{2}} f(x)\right|^{p} d x\right)^{1 / p} d t
$$


Now, we note that

$$
\begin{aligned}
\int_{-1}^{1}|B(\tau t)| & \left(\int_{-\infty}^{\infty}\left|(x+t)^{\alpha+\frac{1}{2}} f(x)\right|^{p} d x\right)^{1 / p} d t \\
\leq & \int_{-1}^{1}|B(\tau t)|\left(\int_{-2}^{2}\left|(x+t)^{\alpha+\frac{1}{2}} f(x)\right|^{p} d x\right)^{1 / p} d t \\
& +\int_{-1}^{1}|B(\tau t)|\left(\int_{|x| \geq 2}\left(\frac{|x|^{\left|\alpha+\frac{1}{2}\right|}}{(|x|-1)^{\left|\alpha+\frac{1}{2}\right|}}\right)^{p}\left|x^{\alpha+\frac{1}{2}} f(x)\right|^{p} d x\right)^{1 / p} d t \\
\leq & \int_{-1}^{1}|B(\tau t)|\left(\int_{t-2}^{t+2}\left|x^{\alpha+\frac{1}{2}} f(x-t)\right|^{p} d x\right)^{1 / p} d t \\
& +2 \int_{-1}^{1}|B(\tau t)|\left(\int_{|x| \geq 2}\left|x^{\alpha+\frac{1}{2}} f(x)\right|^{p} d x\right)^{1 / p} d t \\
\leq & \left\{\max _{|u| \leq 1}\left(\int_{-3}^{3}\left|x^{\alpha+\frac{1}{2}} f(x-u)\right|^{p} d x\right)^{1 / p}|B(\tau t)| d t\right.
\end{aligned}
$$

Furthermore,

$$
\begin{aligned}
& \int_{|t| \geq 1}|B(\tau t)|\left(\int_{-\infty}^{\infty}\left|(x+t)^{\alpha+\frac{1}{2}} f(x)\right|^{p} d x\right)^{1 / p} d t \\
& =\int_{|t| \geq 1}|B(\tau t)|\left(\int_{|x+t| \leq \frac{1}{2}}\left|(x+t)^{\alpha+\frac{1}{2}} f(x)\right|^{p} d x\right. \\
& \left.+\int_{\mathbb{R} \backslash\left[-t-\frac{1}{2},-t+\frac{1}{2}\right]}\left(\left|\frac{x}{x+t}\right|^{\left|\alpha+\frac{1}{2}\right|}|x|^{\alpha+\frac{1}{2}}|f(x)|\right)^{p} d x\right)^{1 / p} d t \\
& \leq \int_{|t| \geq 1}|B(\tau t)|\left(\int_{-\frac{1}{2}}^{\frac{1}{2}}\left|x^{\alpha+\frac{1}{2}} f(x-t)\right|^{p} d x\right. \\
& \left.+(2|t|+1)^{p\left|\alpha+\frac{1}{2}\right|} \int_{-\infty}^{\infty}\left|x^{\alpha+\frac{1}{2}} f(x)\right|^{p} d x\right)^{1 / p} d t \\
& \leq \int_{|t| \geq 1}|B(\tau t)|\left\{\left(\int_{-3}^{3}\left|x^{\alpha+\frac{1}{2}} f(x-t)\right|^{p} d x\right)^{1 / p}\right. \\
& \left.+(2|t|+1)^{\left|\alpha+\frac{1}{2}\right|}\left(\int_{-\infty}^{\infty}\left|x^{\alpha+\frac{1}{2}} f(x)\right|^{p} d x\right)^{1 / p}\right\} d t \\
& \leq \max _{|u| \geq 1}\left(\int_{-3}^{3}\left|x^{\alpha+\frac{1}{2}} f(x-u)\right|^{p} d x\right)^{1 / p} \int_{|t| \geq 1}|B(\tau t)| d t \\
& +\left(\int_{-\infty}^{\infty}\left|x^{\alpha+\frac{1}{2}} f(x)\right|^{p} d x\right)^{1 / p} \int_{|t| \geq 1}(2|t|+1)^{\left|\alpha+\frac{1}{2}\right|}|B(\tau t)| d t .
\end{aligned}
$$

The estimates (80)-(82) imply (78).

Lemma 17. If $f: \mathbb{R} \rightarrow \mathbb{C}$ is measurable and $f(x) /(|x|+1)^{\alpha+\frac{3}{2}} \in L^{p}(\mathbb{R})$ for some $\alpha>-1$ and some $p>1$, then $S_{\tau, \alpha}(f ; \cdot)$ is an entire function. Furthermore, if $\alpha \geq-\frac{1}{2}$, 
$|x|^{\alpha+\frac{1}{2}} f(x) \in L^{p}(\mathbb{R})$, and $f \in L^{p}(-1,1)$, then $S_{\tau, \alpha}(f ; \cdot)$ is of exponential type $\tau$. The same can be said if $-1<\alpha<-\frac{1}{2}, 1<p<\frac{2}{|2 \alpha+1|}$, and $f \in \mathcal{F}^{\alpha, p}(\delta)$ for some $\delta>0$.

Proof. Let

$$
K_{\alpha}(w):=\frac{\tau}{A} \cdot \frac{G_{\alpha}(\tau w)}{(\tau w)^{2}-j_{1}^{2}},
$$

which is an entire function of exponential type $\tau$. Then,

$$
S_{\tau, \alpha}(f ; z)=\int_{-\infty}^{\infty} f(\xi) K_{\alpha}(z-\xi) d \xi .
$$

Now, consider the function

$$
s_{\tau, \alpha}(z)=s_{\tau, \alpha}(z ; a, b):=\int_{a}^{b} f(\xi) K_{\alpha}(z-\xi) d \xi
$$

where $-\infty<a<b<\infty$. Take any point $z \in \mathbb{C}$ and choose $R:=2+\max \{|z-a|$, $|z-b|\}$. The function $K_{\alpha}$ is entire, so there exists a constant $M_{R}$ such that $\left|K_{\alpha}(w)\right| \leq$ $M_{R}$ on $|w|=R$. Hence, for $|h| \leq 1$, we have [8, pp. 72-73]

$$
\begin{aligned}
& \left|\frac{s_{\tau, \alpha}(z+h)-s_{\tau, \alpha}(z)}{h}-\int_{a}^{b} f(\xi) K_{\alpha}^{\prime}(z-\xi) d \xi\right| \\
& =\left|\int_{a}^{b} f(\xi)\left(\frac{K_{\alpha}(z-\xi+h)-K_{\alpha}(z-\xi)}{h}-K_{\alpha}^{\prime}(z-\xi)\right) d \xi\right| \\
& =\left|\frac{h}{2 \pi i} \int_{a}^{b} f(\xi)\left(\int_{|w|=R} \frac{K_{\alpha}(w)}{(w-z+\xi)^{2}(w-z+\xi-h)} d w\right) d \xi\right| \\
& \leq|h| M_{R} R \int_{a}^{b}|f(\xi)| d \xi
\end{aligned}
$$

which tends to zero as $h \rightarrow 0$ since, clearly, $f \in L^{1}(a, b)$. Hence, $s_{\tau, \alpha}(\cdot ; a, b)$ is entire.

Consider the sequence $\left\{\Psi_{n}\right\}_{n \in \mathbb{N}}$ where

$$
\Psi_{n}(z):=\int_{0}^{n} f(\xi) K_{\alpha}(z-\xi) d \xi
$$

Note that for any given $z, K_{\alpha}(z-\zeta)$ is an entire function of exponential type $\tau$ in the variable $\zeta$. From the asymptotic formula (11) for $J_{\alpha}$, it follows that, if $L$ is any given positive number, then for some constant $C_{L}$ depending only on $L$,

$$
\left|K_{\alpha}(x-\xi)\right| \leq \frac{C_{L}}{(|\xi|+1)^{\alpha+\frac{5}{2}}}
$$

for all $x \in[-L, L], \xi \in \mathbb{R}$. The function $F(\zeta):=(\zeta+i)^{\alpha+\frac{5}{2}} K_{\alpha}(x-\zeta)$ is holomorphic and of exponential type $\tau$ in the upper half plane. Besides, $|F(\xi)| \leq C_{L}$ for all $x \in[-L, L], \xi \in \mathbb{R}$. Hence [5, Theorem 6.2.4],

$$
|F(\xi+i y)| \leq C_{L} e^{\tau y}, \quad x \in[-L, L], \quad \xi \in \mathbb{R}, \quad 0 \leq y<\infty,
$$

i.e., for $x \in[-L, L], y \leq 0$, and all $\xi \in \mathbb{R}$, we have

$$
\left|K_{\alpha}(x+i y-\xi)\right| \leq \frac{C_{L} e^{\tau|y|}}{|\xi+i(1+|y|)|^{\alpha+\frac{5}{2}}} .
$$


Clearly, the same estimate also holds for $x \in[-L, L], \xi \in \mathbb{R}, y>0$. Hence, there exists a constant $C_{L}^{*}$ such that

$$
\left|K_{\alpha}(z-\xi)\right| \leq \frac{C_{L}^{*}}{(|\xi|+1)^{\alpha+\frac{5}{2}}} \quad \text { for } \quad|\mathfrak{R e} z| \leq L, \quad|\mathfrak{I m} z| \leq L, \quad \xi \in \mathbb{R}
$$

Since $f(\xi) /(|\xi|+1)^{\alpha+3 / 2} \in L^{p}(\mathbb{R})$, it follows from Hölder's inequality that $\int_{0}^{\infty} f(\xi) K_{\alpha}(z-\xi) d \xi$ is convergent and defines a function $\Psi$. Given any compact set $E \subset \mathbb{C}$, we can find $L>0$ such that $E \subset\{z=x+i y:-L \leq x, y \leq L\}$, so (84) holds for all $z \in E$ and all $\xi \in \mathbb{R}$. This allows us to conclude that $\Psi_{n}(z) \rightarrow \Psi(z)$ uniformly on $E$. The functions $\Psi_{n}$ being all entire, the same can be said about $\Psi(z):=\int_{0}^{\infty} f(\xi) K_{\alpha}(z-\xi) d \xi$. Similarly, $\int_{-\infty}^{0} f(\xi) K_{\alpha}(z-\xi) d \xi$ defines an entire function, so $S_{\tau, \alpha}(f ; \cdot)$ must be entire also.

Now, let $\alpha \geq-\frac{1}{2}$ and suppose that for some $p>1,|x|^{\alpha+\frac{1}{2}} f(x) \in L^{p}(\mathbb{R})$ and $f \in L^{p}(-1,1)$. Then, $f \in L^{p}(\mathbb{R})$. Note that $K_{\alpha}(w) \in L^{q}(\mathbb{R})$ for all $q>\frac{2}{3}$. Hence, for any fixed $z=x+i y$, Hölder's inequality gives $\left(q:=\frac{p}{p-1}\right)$

$$
\begin{aligned}
\left|S_{\tau, \alpha}(f ; z)\right| & =\left|\int_{-\infty}^{\infty} f(\xi) K_{\alpha}(x-\xi+i y) d \xi\right| \\
& \leq\left(\int_{-\infty}^{\infty}|f(\xi)|^{p} d \xi\right)^{1 / p}\left(\int_{-\infty}^{\infty}\left|K_{\alpha}(\xi+i y)\right|^{q} d \xi\right)^{1 / q} \\
& \leq\left(\int_{-\infty}^{\infty}|f(\xi)|^{p} d \xi\right)^{1 / p}\left(\int_{-\infty}^{\infty}\left|K_{\alpha}(\xi)\right|^{q} d \xi\right)^{1 / q} e^{\tau|y|}
\end{aligned}
$$

by Lemma 4 , i.e., $S_{\tau, \alpha}(f ; \cdot)$ is of exponential type $\tau$.

Finally, let $-1<\alpha<-\frac{1}{2}, 1<p<\frac{2}{|2 \alpha+1|}$, and $f \in \mathcal{F}^{\alpha, p}(\delta)$ for some $\delta>0$. Then, for $|x| \rightarrow \infty$,

$$
f(x)=O\left(\frac{1}{(|x|+1)^{\Delta}}\right)
$$

where $\Delta:=\alpha+\frac{1}{2}+\frac{1}{p}+\delta>0$. Hence, $f \in L^{\rho}(\mathbb{R})$ for all $\rho>\frac{1}{\Delta}$. Choose $\rho>\max \left\{1, \frac{1}{\Delta}\right\}$. Then for any fixed $z=x+i y$

$$
\left|S_{\tau, \alpha}(f ; z)\right| \leq\left(\int_{-\infty}^{\infty}|f(\xi)|^{\rho} d \xi\right)^{1 / \rho}\left(\int_{-\infty}^{\infty}\left|K_{\alpha}(\xi+i y)\right|^{q} d \xi\right)^{1 / q}
$$

where $q:=\frac{\rho}{\rho-1}$, from which it follows that $S_{\tau, \alpha}(f ; \cdot)$ is of exponential type $\tau$, as above.

Lemma 18. If $\alpha \geq-\frac{1}{2},|x|^{\alpha+\frac{1}{2}} f(x) \in L^{p}(\mathbb{R})$ for some $p>1$, and $f \in L^{p}(-1,1)$, then

$$
\lim _{\tau \rightarrow \infty} \int_{-\infty}^{\infty}\left|x^{\alpha+\frac{1}{2}}\left(f(x)-S_{\tau, \alpha}(f ; x)\right)\right|^{p} d x=0
$$

The same conclusion also holds when $\alpha \in\left(-1,-\frac{1}{2}\right)$ and $p \in\left(1, \frac{2}{|2 \alpha+1|}\right)$ if $f \in \mathcal{F}^{\alpha, p}(\delta)$ for some $\delta>0$. 
Proof. First, we prove (85) for the characteristic function $\chi$ of the interval $[0,1]$. Clearly,

$$
\begin{aligned}
S_{\tau, \alpha}(\chi ; x) & =\frac{\tau}{A} \int_{-x}^{-x+1} \frac{G_{\alpha}(\tau t)}{(\tau t)^{2}-j_{1}^{2}} d t \\
& =\frac{1}{A} \int_{0}^{\tau x} \frac{G_{\alpha}(t)}{t^{2}-j_{1}^{2}} d t+\frac{1}{A} \int_{0}^{\tau(1-x)} \frac{G_{\alpha}(t)}{t^{2}-j_{1}^{2}} d t
\end{aligned}
$$

There exists a constant $\gamma_{\alpha}$ such that $\left|J_{\alpha}(t)\right| \leq \gamma_{\alpha}$ if $|t| \geq j_{1}$. Hence, for $x \geq j_{1}+1$ and $\tau>1$, we have

$$
\begin{aligned}
\left|\chi(x)-S_{\tau, \alpha}(\chi ; x)\right| & =\left|S_{\tau, \alpha}(\chi ; x)\right| \\
& =\frac{1}{|A|} \int_{\tau(x-1)}^{\tau x} \frac{G_{\alpha}(t)}{t^{2}-j_{1}^{2}} d t \\
& \leq \frac{\gamma_{\alpha}}{|A|} \cdot \frac{1}{(\tau(x-1))^{\alpha}\left(\tau(x-1)-j_{1}\right)} \int_{\tau(x-1)}^{\tau x} \frac{1}{t+j_{1}} d t \\
& \leq \frac{\gamma_{\alpha}}{|A|} \cdot \frac{\tau}{(\tau(x-1))^{\alpha}\left((\tau(x-1))^{2}-j_{1}^{2}\right)} .
\end{aligned}
$$

Given $\varepsilon>0$, there exists, therefore, a number $Y_{\alpha, 1}(\varepsilon) \geq j_{1}+1$ such that

$$
\int_{X}^{\infty}\left|x^{\alpha+\frac{1}{2}}\left(\chi(x)-S_{\tau, \alpha}(\chi ; x)\right)\right|^{p} d x<\frac{\varepsilon}{4}
$$

if $X \geq Y_{\alpha, 1}(\varepsilon)$. Similarly, there exists a number $Y_{\alpha, 2}(\varepsilon) \geq j_{1}+1$ such that

$$
\int_{-\infty}^{-X}\left|x^{\alpha+\frac{1}{2}}\left(\chi(x)-S_{\tau, \alpha}(\chi ; x)\right)\right|^{p} d x<\frac{\varepsilon}{4}
$$

if $X \geq Y_{\alpha, 2}(\varepsilon)$. Let $Y_{\alpha}:=\max \left\{Y_{\alpha, 1}, Y_{\alpha, 2}\right\}$.

From the definition of $A$, it follows that for every $\delta>0$, there exists a positive number $T_{0}(\delta)$ such that

$$
\left|\frac{1}{A} \int_{0}^{T} \frac{G_{\alpha}(t)}{t^{2}-j_{1}^{2}} d t-\frac{1}{2}\right|<\frac{\delta}{2} \quad \text { for all } T>T_{0}(\delta) .
$$

Since

$$
\frac{1}{A} \int_{0}^{u} \frac{G_{\alpha}(t)}{t^{2}-j_{1}^{2}} d t
$$

is a continuous function of $u$ which tends to $\frac{1}{2}$ as $u \rightarrow \infty$, it follows that for all $u \in \mathbb{R}$,

$$
\left|\frac{1}{A} \int_{0}^{u} \frac{G_{\alpha}(t)}{t^{2}-j_{1}^{2}} d t\right| \leq \mu_{\alpha}
$$

where $\mu_{\alpha}$ is a constant depending only on $\alpha$. 
Now, let $\eta$ be any given positive number not exceeding $\frac{1}{2}$ such that

$$
\left(\int_{-\eta}^{\eta}+\int_{1-\eta}^{1+\eta}\right)\left|x^{\alpha+\frac{1}{2}}\right|^{p} d x<\frac{\varepsilon}{4\left(1+2 \mu_{\alpha}\right)^{p}}
$$

Then, for $x \in[\eta, 1-\eta]$ and $\tau>\frac{1}{\eta} T_{0}(\delta)$, both $\tau x$ and $\tau(1-x)$ are larger than $T_{0}(\delta)$, so

$$
\left|S_{\tau, \alpha}(\chi ; x)-1\right| \leq\left|\frac{1}{A} \int_{0}^{\tau x} \frac{G_{\alpha}(t)}{t^{2}-j_{1}^{2}} d t-\frac{1}{2}\right|+\left|\frac{1}{A} \int_{0}^{\tau(1-x)} \frac{G_{\alpha}(t)}{t^{2}-j_{1}^{2}} d t-\frac{1}{2}\right|<\delta .
$$

Similarly, if $x \geq 1+\eta$ or if $x \leq-\eta$, then for $\tau>\frac{1}{\eta} T_{0}(\delta)$

$$
\left|S_{\tau, \alpha}(\chi ; x)\right| \leq\left|\frac{1}{A} \int_{0}^{\tau|x|} \frac{G_{\alpha}(t)}{t^{2}-j_{1}^{2}} d t-\frac{1}{2}\right|+\left|\frac{1}{A} \int_{0}^{\tau|1-x|} \frac{G_{\alpha}(t)}{t^{2}-j_{1}^{2}} d t-\frac{1}{2}\right|<\delta .
$$

Thus, if $E_{\eta}:=\{x:|x|<\eta$ or $|x-1|<\eta\}$, then

$$
\left|\chi(x)-S_{\tau, \alpha}(\chi ; x)\right|<\delta \quad \text { for all } x \in \mathbb{R} \backslash E_{\eta}
$$

if $\tau>\frac{1}{\eta} T_{0}(\delta)$. Now, setting

$$
\delta:=\left(i \frac{\varepsilon}{4 \int_{-Y_{\alpha}}^{Y_{\alpha}}\left|x^{\alpha+\frac{1}{2}}\right|^{p} d x}\right)^{1 / p}
$$

we obtain

$$
\int_{\left[-Y_{\alpha}, Y_{\alpha}\right] \backslash E_{\eta}}\left|x^{\alpha+\frac{1}{2}}\left(\chi(x)-S_{\tau, \alpha}(\chi ; x)\right)\right|^{p} d x<\delta^{p} \cdot \int_{-Y_{\alpha}}^{Y_{\alpha}}\left|x^{\alpha+\frac{1}{2}}\right|^{p} d x=\frac{\varepsilon}{4}
$$

for all $\tau>\frac{1}{\eta} T_{0}(\delta)=: T_{1}(\varepsilon)$. Furthermore,

$$
\int_{E_{\eta}}\left|x^{\alpha+\frac{1}{2}}\left(\chi(x)-S_{\tau, \alpha}(\chi ; x)\right)\right|^{p} d x \leq\left(1+2 \mu_{\alpha}\right)^{p} \int_{E_{\eta}}\left|x^{\alpha+\frac{1}{2}}\right|^{p} d x<\frac{\varepsilon}{4} .
$$

It follows from (86)-(89) that (85) holds for the characteristic function on $[0,1]$. A similar argument applies to the characteristic function on $[1,2]$. The result then easily extends to the characteristic function of any finite interval, and indeed to any step function with compact support.

Now, let $\alpha \geq-\frac{1}{2}$ and $|x|^{\alpha+\frac{1}{2}} f(x) \in L^{p}(\mathbb{R})$ for some $p>1$. Furthermore, let $f \in L^{p}(-1,1)$. Given $\varepsilon>0$, there exists, by Lemma 15, a step function $\Omega$ with compact support such that (68) holds. As explained above, it is possible to choose $\tau_{1}$ such that for all $\tau>\tau_{1}$, we have

$$
\left(\int_{-\infty}^{\infty}\left|x^{\alpha+\frac{1}{2}}\left(\Omega(x)-S_{\tau, \alpha}(\Omega ; x)\right)\right|^{p} d x\right)^{1 / p}<\varepsilon .
$$


Now, we note that

$$
\begin{aligned}
& \left(\int_{-\infty}^{\infty}\left|x^{\alpha+\frac{1}{2}}\left(f(x)-S_{\tau, \alpha}(f ; x)\right)\right|^{p} d x\right)^{1 / p} \\
& \leq\left(\int_{-\infty}^{\infty}\left|x^{\alpha+\frac{1}{2}}\left(\Omega(x)-S_{\tau, \alpha}(f ; x)\right)\right|^{p} d x\right)^{1 / p}+\left(\int_{-\infty}^{\infty}\left|x^{\alpha+\frac{1}{2}}(f(x)-\Omega(x))\right|^{p} d x\right)^{1 / p} \\
& \leq\left(\int_{-\infty}^{\infty}\left|x^{\alpha+\frac{1}{2}} S_{\tau, \alpha}(f-\Omega ; x)\right|^{p} d x\right)^{1 / p}+\left(\int_{-\infty}^{\infty}\left|x^{\alpha+\frac{1}{2}}\left(\Omega(x)-S_{\tau, \alpha}(\Omega ; x)\right)\right|^{p} d x\right)^{1 / p} \\
& \quad+\left(\int_{-\infty}^{\infty}\left|x^{\alpha+\frac{1}{2}}(f(x)-\Omega(x))\right|^{p} d x\right)^{1 / p} \\
& \leq C_{\alpha, p}\left\{\left(\int_{-1}^{1}|f(x)-\Omega(x)|^{p} d x\right)^{1 / p}+\left(\int_{|x| \geq 1}\left|x^{\alpha+\frac{1}{2}}(f(x)-\Omega(x))\right|^{p} d x\right)^{1 / p}\right\} \\
& \quad+\left(\int_{-\infty}^{\infty}\left|x^{\alpha+\frac{1}{2}}\left(\Omega(x)-S_{\tau, \alpha}(\Omega ; x)\right)\right|^{p} d x\right)^{1 / p} \\
& \quad+\left(\int_{-\infty}^{\infty}\left|x^{\alpha+\frac{1}{2}}(f(x)-\Omega(x))\right|^{p} d x\right)^{1 / p}
\end{aligned}
$$

since $f-\Omega$ clearly satisfies the conditions of part (i) of Lemma 16. By (68) and (90), we conclude that

$$
\left(\int_{-\infty}^{\infty}\left|x^{\alpha+\frac{1}{2}}\left(f(x)-S_{\tau, \alpha}(f ; x)\right)\right|^{p} d x\right)^{1 / p} \leq C_{\alpha, p} \cdot 2 \varepsilon+\varepsilon+2 \varepsilon .
$$

Since $\varepsilon$ is arbitrary, (85) holds for the case under consideration.

Finally, let $-1<\alpha<-\frac{1}{2}, 1<p<\frac{2}{|2 \alpha+1|}$, and $f \in \mathcal{F}^{\alpha, p}(\delta)$ for some $\delta>0$. This time, by Lemma 15, there exists a step function $\Omega$ with compact support such that (69) holds. Since $f-\Omega$ satisfies the conditions of part (ii) of Lemma 16, using (78), we obtain

$$
\begin{aligned}
& \left(\int_{-\infty}^{\infty}\left|x^{\alpha+\frac{1}{2}}\left(f(x)-S_{\tau, \alpha}(f ; x)\right)\right|^{p} d x\right)^{1 / p} \\
& \leq C_{\alpha, p}^{\prime}\left\{\left(\int_{-1}^{1}\left|x^{\alpha+\frac{1}{2}}(f(x)-\Omega(x))\right|^{p} d x\right)^{1 / p}+\left(\int_{|x| \geq 1}\left|x^{\alpha+\frac{1}{2}}(f(x)-\Omega(x))\right|^{p} d x\right)^{1 / p}\right. \\
& \left.\quad+\max _{u \in \mathbb{R}}\left(\int_{-3}^{3}\left|x^{\alpha+\frac{1}{2}}(f(x-u)-\Omega(x-u))\right|^{p} d x\right)^{1 / p}\right\} \\
& +\left(\int_{-\infty}^{\infty}\left|x^{\alpha+\frac{1}{2}}\left(\Omega(x)-S_{\tau, \alpha}(\Omega ; x)\right)\right|^{p} d x\right)^{1 / p} \\
& +\left(\int_{-\infty}^{\infty}\left|x^{\alpha+\frac{1}{2}}(f(x)-\Omega(x))\right|^{p} d x\right)^{1 / p} .
\end{aligned}
$$

By (69) and (90), we get

$$
\left(\int_{-\infty}^{\infty}\left|x^{\alpha+\frac{1}{2}}\left(f(x)-S_{\tau, \alpha}(f ; x)\right)\right|^{p} d x\right)^{1 / p} \leq C_{\alpha, p}^{\prime} \cdot 3 \varepsilon+\varepsilon+2 \varepsilon,
$$

which completes the proof of Lemma 18.

Remark 9. Lemma 18 seems to be a result of independent interest. 
Lemma 19. According as $\alpha$ belongs to $\left[-\frac{1}{2}, \infty\right]$ or to $\left(-1,-\frac{1}{2}\right)$, let $f$ be in $\mathcal{F}^{\alpha, p} \cap \mathcal{R}$ for some $p$ in $(1, \infty)$ or in $\left(1, \frac{2}{|2 \alpha+1|}\right)$, respectively. If $f^{*}(x):=f(x)-S_{\sigma, \alpha}(f ; x)$ where $\sigma>0$, then

$$
\lim _{\tau \rightarrow \infty} \sum_{\substack{k=-\infty \\ k \neq 0}}^{\infty} \frac{j_{k}-j_{k-1}}{\tau}\left|\left(\frac{j_{k}}{\tau}\right)^{\alpha+\frac{1}{2}} f^{*}\left(\frac{j_{k}}{\tau}\right)\right|^{p}=\int_{-\infty}^{\infty}\left|x^{\alpha+\frac{1}{2}} f^{*}(x)\right|^{p} d x
$$

Proof. By Lemma 17, the function $S_{\sigma, \alpha}(f ; \cdot)$ is entire and of exponential type. By Lemma $16, x^{\alpha+\frac{1}{2}} S_{\sigma, \alpha}(f ; x)$ belongs to $L^{p}(\mathbb{R})$ and, therefore to $L^{p}(0, \infty)$. Since $W(z)$ $:=z^{\alpha+\frac{1}{2}} S_{\sigma, \alpha}(f ; z)$ is holomorphic and of exponential type in the open right half-plane, it follows from Lemma 8 that $W^{\prime} \in L^{p}[a, \infty)$ for every $a>0$.

Let $C$ be such that

$$
\left|x^{\alpha+\frac{1}{2}} f(x)\right|<\frac{C}{(|x|+1)^{\delta+\frac{1}{p}}} \quad \text { for some } \delta>0 \text { and all } x \in \mathbb{R} .
$$

Given $\varepsilon>0$, we choose $X_{\varepsilon}$ in $\left[\left(12 C^{p} / \delta p \varepsilon\right)^{1 / \delta p}, \infty\right)$ large enough to have

$$
\begin{gathered}
\int_{|x| \geq X_{\varepsilon}}\left|x^{\alpha+\frac{1}{2}} f(x)\right|^{p} d x<\frac{\varepsilon}{12}, \\
\int_{|x| \geq X_{\varepsilon}}|W(x)|^{p} d x<\frac{1}{2^{p-1}} \cdot \frac{\varepsilon}{24}, \\
\int_{|x| \geq X_{\varepsilon}}\left|W^{\prime}(x)\right|^{p} d x<\frac{1}{2^{p-1}} \cdot \frac{\varepsilon}{24} .
\end{gathered}
$$

If $l=l(\tau)$ is the largest integer such that $j_{l} / \tau \leq X_{\varepsilon}$, then

$$
\begin{gathered}
\sum_{k=l+2}^{\infty} \frac{j_{k}-j_{k-1}}{\tau}\left|\left(\frac{j_{k}}{\tau}\right)^{\alpha+\frac{1}{2}} f\left(\frac{j_{k}}{\tau}\right)\right|^{p}<\frac{C^{p}}{\tau} \sum_{k=l+2}^{\infty} \frac{j_{k}-j_{k-1}}{\left(j_{k} / \tau\right)^{1+\delta p}} \\
<C^{p} \tau^{\delta p} \int_{j_{l+1}}^{\infty} \frac{1}{x^{1+\delta p}} d x=\frac{C^{p}}{\delta p} \cdot\left(\frac{\tau}{j_{l+1}}\right)^{\delta p}<\frac{\varepsilon}{12}
\end{gathered}
$$

since $\left(\tau / j_{l+1}\right)^{\delta p}<\left(1 / X_{\varepsilon}\right)^{\delta p}<\delta p \varepsilon / 12 C^{p}$. Similarly,

$$
\sum_{k=-\infty}^{-l-2} \frac{j_{k}-j_{k-1}}{\tau}\left|\left(\frac{j_{k}}{\tau}\right)^{\alpha+\frac{1}{2}} f\left(\frac{j_{k}}{\tau}\right)\right|^{p}<\frac{\varepsilon}{12} .
$$

For each $k \in \mathbb{Z}$, let $\xi_{k} \in\left[j_{k-1} / \tau, j_{k} / \tau\right]$ be such that

$$
\int_{\frac{j_{k-1}}{\tau}}^{\frac{j_{k}}{\tau}}|W(x)|^{p} d x=\frac{j_{k}-j_{k-1}}{\tau} \cdot\left|W\left(\xi_{k}\right)\right|^{p} .
$$

Then

$$
\begin{aligned}
& \sum_{k=l+2}^{\infty} \frac{j_{k}-j_{k-1}}{\tau}\left|W\left(\frac{j_{k}}{\tau}\right)\right|^{p}=\sum_{k=l+2}^{\infty} \frac{j_{k}-j_{k-1}}{\tau}\left|W\left(\frac{j_{k}}{\tau}\right)-W\left(\xi_{k}\right)+W\left(\xi_{k}\right)\right|^{p} \\
& \leq 2^{p-1} \sum_{k=l+2}^{\infty} \frac{j_{k}-j_{k-1}}{\tau}\left(\left|W\left(\frac{j_{k}}{\tau}\right)-W\left(\xi_{k}\right)\right|^{p}+\left|W\left(\xi_{k}\right)\right|^{p}\right) \\
& =2^{p-1} \sum_{k=l+2}^{\infty}\left(\frac{j_{k}-j_{k-1}}{\tau}\left|\int_{\xi_{k}}^{\frac{j_{k}}{\tau}} W^{\prime}(x) d x\right|^{p}+\int_{\frac{j_{k-1}}{\tau}}^{\frac{j_{k}}{\tau}}|W(x)|^{p} d x\right)
\end{aligned}
$$


by (97). Clearly,

$$
\begin{aligned}
\left|\int_{\xi_{k}}^{\frac{j_{k}}{\tau}} W^{\prime}(x) d x\right|^{p} & \leq \int_{\frac{j_{k-1}}{\tau}}^{\frac{j_{k}}{\tau}}\left|W^{\prime}(x)\right|^{p} d x \cdot\left(\int_{\frac{j_{k-1}}{\tau}}^{\frac{j_{k}}{\tau}} 1 d x\right)^{p-1} \\
& =\left(\frac{j_{k}-j_{k-1}}{\tau}\right)^{p-1} \int_{\frac{j_{k-1}}{\tau}}^{\frac{j_{k}}{\tau}}\left|W^{\prime}(x)\right|^{p} d x
\end{aligned}
$$

so

$$
\begin{aligned}
& \sum_{k=l+2}^{\infty} \frac{j_{k}-j_{k-1}}{\tau}\left|W\left(\frac{j_{k}}{\tau}\right)\right|^{p} \\
& \leq 2^{p-1} \sum_{k=l+2}^{\infty}\left(\left(\frac{j_{k}-j_{k-1}}{\tau}\right)^{p} \int_{\frac{j_{k-1}}{\tau}}^{\frac{j_{k}}{\tau}}\left|W^{\prime}(x)\right|^{p} d x+\int_{\frac{j_{k-1}}{\tau}}^{\frac{j_{k}}{\tau}}|W(x)|^{p} d x\right) \\
& \leq 2^{p-1}\left(\left(\frac{\delta_{2}}{\tau}\right)^{p} \int_{\frac{j_{l+1}}{\tau}}^{\infty}\left|W^{\prime}(x)\right|^{p} d x+\int_{\frac{j_{l+1}}{\tau}}^{\infty}|W(x)|^{p} d x\right) \quad[\text { by }(16)] \\
& \leq 2^{p-1}\left(\left(\frac{\delta_{2}}{\tau}\right)^{p} \int_{X_{\varepsilon}}^{\infty}\left|W^{\prime}(x)\right|^{p} d x+\int_{X_{\varepsilon}}^{\infty}|W(x)|^{p} d x\right)<\frac{\varepsilon}{12}
\end{aligned}
$$

by (93) and (94), if $\tau>\delta_{2}$. Similarly,

$$
\sum_{k=-\infty}^{-l-2} \frac{j_{k}-j_{k-1}}{\tau}\left|W\left(\frac{j_{k}}{\tau}\right)\right|^{p}<\frac{\varepsilon}{12} \quad \text { if } \tau>\delta_{2} .
$$

It is clear that $\left|x^{\alpha+\frac{1}{2}} f(x)\right|^{p}$ and $|W(x)|^{p}$ both belong to $\mathcal{R}$. Hence, taking note of (92) and (93), we can claim that

$$
\begin{aligned}
\left.\left|\sum_{k=-l-1}^{l+1} \frac{j_{k}-j_{k-1}}{\tau}\right|\left(\frac{j_{k}}{\tau}\right)^{\alpha+\frac{1}{2}}\left(f\left(\frac{j_{k}}{\tau}\right)-S_{\sigma, \alpha}\left(f ; \frac{j_{k}}{\tau}\right)\right)\right|^{p} \\
-\int_{-X_{\varepsilon}}^{X_{\varepsilon}}\left|x^{\alpha+\frac{1}{2}}\left(f(x)-S_{\sigma, \alpha}(f ; x)\right)\right|^{p} d x \mid<\frac{7 \varepsilon}{12}
\end{aligned}
$$

for all large $\tau$. The desired result follows from (92), (93), (95), (96), and (98)-(100).

3.2. Proof of Theorem 1. Let $\sigma>0$, and consider

$$
f_{\sigma}^{*}:=f-S_{\sigma, \alpha}(f ; \cdot) .
$$

By Lemma $17, S_{\sigma, \alpha}(f ; \cdot)$ is an entire function of exponential type $\sigma$ and so $\left(x^{\alpha+\frac{1}{2}} S_{\sigma, \alpha}(f ; x)\right)^{p}$ is regular and of exponential type in the open right half-plane. Furthermore, by Lemma $16, \int_{-\infty}^{\infty}\left|x^{\alpha+\frac{1}{2}} S_{\sigma, \alpha}(f ; x)\right|^{p} d x<\infty$. Hence, by Lemma 5 ,

$$
\sum_{\substack{k=-\infty \\ k \neq 0}}^{\infty}\left|\left(\frac{j_{k}}{\tau}\right)^{\alpha+\frac{1}{2}} S_{\sigma, \alpha}\left(f ; \frac{j_{k}}{\tau}\right)\right|^{p}<\infty, \quad \tau>0 .
$$


Since the function $f$ obviously satisfies (39), it follows from (101) and Lemma 12 that the series for $L_{\tau, \alpha}\left(f_{\sigma}^{*}+S_{\sigma, \alpha}(f ; \cdot) ; z\right)$ is absolutely convergent. Hence, for $\tau \geq \sigma$,

$$
\begin{aligned}
L_{\tau, \alpha}(f ; z) & =L_{\tau, \alpha}\left(f_{\sigma}^{*}+S_{\sigma, \alpha}(f ; \cdot) ; z\right) \\
& =L_{\tau, \alpha}\left(f_{\sigma}^{*} ; z\right)+L_{\tau, \alpha}\left(S_{\sigma, \alpha}(f ; \cdot) ; z\right) \\
& =L_{\tau, \alpha}\left(f_{\sigma}^{*} ; z\right)+S_{\sigma, \alpha}(f ; z)
\end{aligned}
$$

by Lemma 13. Therefore,

$$
f(z)-L_{\tau, \alpha}(f ; z)=f_{\sigma}^{*}(z)-L_{\tau, \alpha}\left(f_{\sigma}^{*} ; z\right)
$$

and

$$
\begin{aligned}
& \left(\int_{-\infty}^{\infty}\left|x^{\alpha+\frac{1}{2}}\left(f_{\sigma}^{*}(x)-L_{\tau, \alpha}\left(f_{\sigma}^{*} ; x\right)\right)\right|^{p} d x\right)^{1 / p} \\
& \left.\leq 2^{(p-1) / p}\left\{\left(\int_{-\infty}^{\infty}\left|x^{\alpha+\frac{1}{2}} f_{\sigma}^{*}(x)\right|^{p} d x\right)^{1 / p}+\left.\left(\int_{-\infty}^{\infty} \mid x^{\alpha+\frac{1}{2}} L_{\tau, \alpha}\left(f_{\sigma}^{*} ; x\right)\right)\right|^{p} d x\right)^{1 / p}\right\} .
\end{aligned}
$$

In view of the hypothesis on $f$ and (101), we have

$$
\sum_{\substack{k=-\infty \\ k \neq 0}}^{\infty}\left|\left(\frac{j_{k}}{\tau}\right)^{\alpha+\frac{1}{2}} f_{\sigma}^{*}\left(\frac{j_{k}}{\tau}\right)\right|^{p}<\infty
$$

so, by Lemma 14,

$$
\begin{aligned}
\left.\int_{-\infty}^{\infty} \mid x^{\alpha+\frac{1}{2}} L_{\tau, \alpha}\left(f_{\sigma}^{*} ; x\right)\right)\left.\right|^{p} d x & \leq B_{\alpha, p}^{p} \frac{\pi}{\tau} \sum_{\substack{k=-\infty \\
k \neq 0}}^{\infty}\left|\frac{1}{\tau^{\alpha+\frac{1}{2}} G_{\alpha}^{\prime}\left(j_{k}\right)} \cdot f_{\sigma}^{*}\left(\frac{j_{k}}{\tau}\right)\right|^{p} \\
& \leq B_{\alpha, p}^{p} \frac{\pi}{\delta_{1} c_{2}} \sum_{\substack{k=-\infty \\
k \neq 0}}^{\infty} \frac{j_{k}-j_{k-1}}{\tau}\left|\left(\frac{j_{k}}{\tau}\right)^{\alpha+\frac{1}{2}} f_{\sigma}^{*}\left(\frac{j_{k}}{\tau}\right)\right|^{p}
\end{aligned}
$$

by (19) and (15). Now, it follows from Lemma 19 that

$$
\left.\lim _{\tau \rightarrow \infty} \int_{-\infty}^{\infty} \mid x^{\alpha+\frac{1}{2}} L_{\tau, \alpha}\left(f_{\sigma}^{*} ; x\right)\right)\left.\right|^{p} d x \leq B_{\alpha, p}^{p} \frac{\pi}{\delta_{1} c_{2}} \int_{-\infty}^{\infty}\left|x^{\alpha+\frac{1}{2}} f_{\sigma}^{*}(x)\right|^{p} d x
$$

Hence,

$$
\begin{aligned}
& \lim _{\tau \rightarrow \infty}\left(\int_{-\infty}^{\infty}\left|x^{\alpha+\frac{1}{2}}\left(f(x)-L_{\tau, \alpha}(f ; x)\right)\right|^{p} d x\right)^{1 / p} \\
& =\lim _{\tau \rightarrow \infty}\left(\int_{-\infty}^{\infty}\left|x^{\alpha+\frac{1}{2}}\left(f_{\sigma}^{*}(x)-L_{\tau, \alpha}\left(f_{\sigma}^{*} ; x\right)\right)\right|^{p} d x\right)^{1 / p} \\
& \leq 2^{(p-1) / p}\left\{1+B_{\alpha, p}\left(\frac{\pi}{\delta_{1} c_{2}}\right)^{1 / p}\right\}\left(\int_{-\infty}^{\infty}\left|x^{\alpha+\frac{1}{2}} f_{\sigma}^{*}(x)\right|^{p} d x\right)^{1 / p} .
\end{aligned}
$$

Now let $\varepsilon$ be any positive number. In view of Lemma 18, the right-hand side of (102) can be made less than $\varepsilon$ by taking $\sigma$ large enough. With this, the theorem is proved. 
3.3. Justification of Remark 1. If $X$ denotes the Banach space of all continuous functions $f: \mathbb{R} \rightarrow \mathbb{C}$ which vanish outside $[0,1]$, then $f \rightarrow L_{\tau, \alpha}(f ; \cdot)$ defines a bounded linear transformation $\Lambda_{\tau, \alpha}$ from $X$ to the normed linear space $C[-1,1]$ of all continuous functions $\phi$ with $\|\phi\|:=\max _{-1 \leq x \leq 1}|\phi(x)|$. For all large $\tau$, we have

$$
\begin{aligned}
\left\|\Lambda_{\tau, \alpha}\right\| & \geq \sum_{0<\frac{j_{\nu}}{\tau} \leq 1} \frac{\left|G_{\alpha}\left(\frac{1}{2} j_{1}\right)\right|}{\left|G_{\alpha}^{\prime}\left(j_{\nu}\right)\right|\left|j_{\nu}-\frac{1}{2} j_{1}\right|} \\
& >\frac{1}{c_{2}}\left|G_{\alpha}\left(\frac{1}{2} j_{1}\right)\right| \sum_{0<\frac{j_{\nu}}{\tau} \leq 1} \frac{\left|j_{\nu}\right|^{\alpha+\frac{1}{2}}}{\left|j_{\nu}-\frac{1}{2} j_{1}\right|} \\
& \geq \frac{1}{c_{2}}\left|G_{\alpha}\left(\frac{1}{2} j_{1}\right)\right| \sum_{0<\frac{j_{\nu}}{\tau} \leq 1} \frac{1}{\left|j_{\nu}-\frac{1}{2} j_{1}\right|}
\end{aligned}
$$

since $j_{1} \geq \pi / 2$ for $\alpha \geq-1 / 2$. Using the asymptotic formula (14), we easily conclude that

$$
\left\|\Lambda_{\tau, \alpha}\right\|>c_{11} \log \tau
$$

where $c_{11}=c_{11}(\alpha)$ is a positive constant depending only on $\alpha$. Thus, $\sup _{\tau}\left\|\Lambda_{\tau, \alpha}\right\|=\infty$. Hence, by the Banach-Steinhaus theorem [30, p. 98], there exists a function $f^{*} \in X$ and so satisfying (8) such that

$$
\max _{-1 \leq x \leq 1}\left|f^{*}(x)-L_{\tau, \alpha}\left(f^{*} ; x\right)\right|
$$

does not remain bounded as $\tau \rightarrow \infty$. This idea has been used before in a similar situation [12].

3.4. Justification of Remark 3. For $p \geq 2$, we obviously have

$$
\int_{|x| \geq 1}\left|f(x)-L_{\tau, \alpha}(f ; x)\right|^{p}|x|^{2 \alpha+1} d x \leq \int_{|x| \geq 1}\left|x^{\alpha+\frac{1}{2}}\left(f(x)-L_{\tau, \alpha}(f ; x)\right)\right|^{p} d x,
$$

so it is enough to check that

$$
\int_{-1}^{1}\left|f(x)-L_{\tau, \alpha}(f ; x)\right|^{p}|x|^{2 \alpha+1} d x \rightarrow 0 \quad \text { as } \tau \rightarrow \infty .
$$

Going through the proof of Theorem 1, we need to show that if $\tau>\sigma$ and $\sigma \rightarrow \infty$, then

$$
\begin{gathered}
\int_{-1}^{1}|x|^{2 \alpha+1}\left|f(x)-S_{\sigma, \alpha}(f ; x)\right|^{p} d x \rightarrow 0 \\
\int_{-1}^{1}|x|^{2 \alpha+1}\left|L_{\tau, \alpha}\left(f_{\sigma}^{*} ; x\right)\right|^{p} d x \rightarrow 0 .
\end{gathered}
$$

As regards (104), it can be established in a manner analogous to Lemma 18 by first proving it for step functions, which involves verifying it for $[0, a],[a, b]$ where $0<a<b \leq 1$; the argument can be completed as before with the help of Lemma 15 and the following (easy to prove) modification of Part (i) of Lemma 16. 
Lemma 16'. If $f$ satisfies the conditions of Part (i) of Lemma 16, then for $\tau \geq 1$, we have

$$
\begin{aligned}
\int_{-1}^{1}|x|^{2 \alpha+1}\left|S_{\tau, \alpha}(f ; x)\right|^{p} d x \leq C_{\alpha, p}^{\prime \prime} & \left\{\left(\int_{-1}^{1}|f(x)|^{p} d x\right)^{1 / p}\right. \\
& \left.+\left(\int_{|x| \geq 1}|x|^{2 \alpha+1}|f(x)|^{p} d x\right)^{1 / p}\right\}
\end{aligned}
$$

where $C_{\alpha, p}^{\prime \prime}$ is a constant depending only on $\alpha$ and $p$.

In order to verify (105) we first observe that

$$
\int_{-1}^{1}|x|^{2 \alpha+1}\left|L_{\tau, \alpha}\left(f_{\sigma}^{*} ; x\right)\right|^{p} d x \leq B_{\alpha, p}^{p} \frac{\pi}{\tau} \sum_{\substack{k=-\infty \\ k \neq 0}}^{\infty}\left|\frac{1}{\tau^{\alpha+\frac{1}{2}} G_{\alpha}^{\prime}\left(j_{k}\right)} f_{\sigma}^{*}\left(\frac{j_{k}}{\tau}\right)\right|^{p}
$$

which can be proved the same way as (53). Then we apply Lemmas 19 and 18 as in the proof of Theorem 1 .

Acknowledgment. We were not sure about the originality of Lemma 3, so we consulted Professors R. Askey, L. Lorch, M. Muldoon, and F. W. J. Olver. None of them remembered having seen the result before. We thank them for their interest and some useful comments. The first author gratefully acknowledges the support from the Natural Sciences and Engineering Research Council of Canada Grant No. A-3081 of the second author; he also was partially supported by Grant No. MM-15 from the Bulgarian Ministry of Sciences.

\section{References}

1. M. Abramovitz and I. A. Stegun (Eds.), Handbook of Mathematical Functions: with Formulas, Graphs and Mathematical Tables, Dover Publication, New York, 1965.

2. R. Askey, Mean convergence of orthogonal series and Lagrange interpolation, Acta Math. Acad. Sci. Hungar. 23 (1972), 71-85.

3. _ Summability of Jacobi series, Trans. Amer. Math. Soc. 179 (1973), 71-84.

4. R. P. Boas, Jr., Inequalities between series and integrals involving entire functions, J. Indian Math. Soc. 16 (1952), 127-135.

5. Entire Functions, Academic Press, New York, 1954.

6. R. P. Boas, Jr. and H. Pollard, Complete sets of Bessel and Legendre functions, Ann. of Math. (2) 48 (1947), 366-383.

7. L. L. Campbell, A comparison of the sampling theorems of Kramer and Whittaker, SIAM J. Appl. Math. 12 (1964), 117-130.

8. E. T. Copson, An Introduction to the Theory of Functions of a Complex Variable, Oxford University Press, London, 1935.

9. P. Erdös and P. Turán, On interpolation. I. Quadrature and mean convergence in the Lagrange interpolation, Ann. of Math. (2) 38 (1937), 142-155.

10. G. Faber, Über die interpolatorische Darstellung stetiger Funktionen, Jber. Deutsch. Math. Verein. 23 (1914), 192-210.

11. C. Frappier and P. Olivier, A quadrature formula involving zeros of Bessel functions, Math. Comp. 60 (1993), 303-316.

12. R. Gervais, Q. I. Rahman, and G. Schmeisser, Simultaneous interpolation and approximation. In: Polynomial and Spline Approximation (Proc. NATO Adv. Study Inst., Univ. Calgary, Calgary, Alta, 1978, Badri N. Sahney, ed.) NATO Adv. Study Inst. Ser., Ser. C: Math. and Phys. Sci., Vol 49, Reidel, Dordrecht (1979), pp. 203-223.

13. G. R. Grozev and Q. I. Rahman, A quadrature formula with zeros of Bessel functions as nodes, Math. Comp. 64 (1995), 715-725.

14. G. Grünwald, Über Divergenzerscheinungen der Lagrangeschen Interpolationspolynome stetiger Funktionen, Ann. of Math. (2) 37 (1936), 908-918.

15. A. R. Harvey, The mean of a function of exponential type, Amer. J. Math. 70 (1948), 181-202. 
16. E. Hille, Analytic Function Theory, volume II, Ginn and Company, Boston, New York, 1962.

17. H. P. Kramer, A generalized sampling theorem, J. Math. and Phys. 38 (1959), 68-72.

18. A. J. Macintyre, Laplace's transformation and integral functions, Proc. London Math. Soc. 45 (1938), 1-20.

19. J. Marcinkiewicz, Sur l'interpolation (I), Studia Math. 6 (1936), 1-17.

20. _ Sur la divergence des polynômes d'interpolation, Acta Litter. Sci. Szeged 8 (1937), 131135.

21. W. Marshall, On a new method of computing the roots of Bessel's functions, Ann. of Math. (2) 11 (1910), 153-160.

22. J. McMahon, On the roots of the Bessel and certain related functions, Ann. of Math. 9 (1895), 23-30.

23. P. Nevai, Mean convergence of Lagrange interpolation III, Trans. Amer. Math. Soc. 282 (1984), 669-698.

24. S. M. Nikol'skiŭ, Approximation of Functions of Several Variables and Imbedding Theorems, Springer-Verlag, New York, Berlin, 1975.

25. M. Plancherel and G. Pólya, Fonctions entières et intégrales de Fourier multiples, Comment. Math. Helv. 9 (1937), 224-248; 10 (1938), 110-163.

26. Q. I. Rahman and G. Schmeisser, $L^{p}$ inequalities for entire functions of exponential type, Trans. Amer. Math. Soc. 320 (1990), 91-103.

27. Q. I. Rahman and F. Vértesi, On the $L^{p}$ convergence of Lagrange interpolating entire functions of exponential type, J. Approx. Theory 69 (1992), 302-317.

28. M. D. Rawn, On nonuniform sampling expansions, using entire interpolating functions, and on the stability of Bessel-type sampling expansions, IEEE Trans. Inform. Theory 35 (1989), 549-557.

29. H. L. Royden, Real Analysis, 2nd edition, Macmillan Publishing Co., Inc., New York, 1968.

30. W. Rudin, Real and Complex Analysis, McGraw-Hill, New York, 1966.

31. G. Szegö, Orthogonal Polynomials, 4th edition, American Mathematical Society Colloquium Publications Vol. XXIII, Providence, Rhode Island, 1975.

32. E. C. Titchmarsh, Reciprocal formulae involving series and integrals, Math. Z. 25 (1926), 321347.

33. G. N. Watson, A Treatise on the Theory of Bessel Functions, 2nd edition, Cambridge University Press, Cambridge, 1945.

34. P. Weiss, Sampling theorems associated with Sturm-Liouville systems, Bull. Amer. Math. Soc. 63 (1957), 242.

35. E. T. Whittaker and G. N. Watson, A Course of Modern Analysis, 4th edition, Cambridge University Press, Cambridge, 1940.

Département de Mathématiques et de Statistique, Université de Montréal, Montréal, QuÉBEc, CANADA, H3C 3J7

E-mail: georgig@numetrix.com, rahmanqi@ere.umontreal.ca 